KANAZAWA-10-09

November, 2010

\title{
Dark matter in the supersymmetric radiative seesaw model with an anomalous $\mathrm{U}(1)$ symmetry
}

\author{
Daijiro Suematsu and Takashi Toma2 \\ Institute for Theoretical Physics, Kanazawa University, \\ Kanazawa 920-1192, Japan
}

\begin{abstract}
The existence of an anomalous $\mathrm{U}(1)$ symmetry is shown to play a crucial role in the supersymmetric radiative seesaw model for neutrino masses. It explains the smallness of some couplings related to neutrino mass generation in a favorable way in addition to cause the hierarchical structure of Yukawa couplings of quarks and leptons. If it is spontaneously broken to a $Z_{2}$ subgroup, this $Z_{2}$ symmetry can make a lifetime of the lightest field with its odd parity extremely long. Thus, the model has an additional dark matter candidate other than the lightest neutralino, which appears in the $R$-parity conserved MSSM. We discuss the nature of dark matter by taking account of its relation to the neutrino mass generation and the lepton flavor violation.
\end{abstract}

\footnotetext{
${ }^{1}$ e-mail: suematsu@hep.s.kanazawa-u.ac.jp

2e-mail: t-toma@hep.s.kanazawa-u.ac.jp
} 


\section{Introduction}

The recent astrophysical observations [1] and neutrino oscillation experiments [2] require that the standard model (SM) should be extended so as to include dark matter (DM) [3] and small neutrino masses. A radiative seesaw model proposed by Ma [4] is a simple and interesting possibility among such extensions 3 In this model the existence of DM is intimately related to the neutrino mass generation. The combined study of the neutrino oscillation data, the lepton flavor violating processes such as $\mu \rightarrow e \gamma$ and the DM relic abundance can give strong constraints on the model [5, 6]. On the other hand, the recently reported cosmic ray anomaly [12, 13] attracts a lot of attention as a target of the DM physics. Various work relevant to this has been done on the basis of the annihilation of DM [7, 14, 15, 16, 17] and also the decay of DM [18, 19, 20, 21, 22]. If we impose that this neutrino mass model should explain this anomaly, it could bring the model additional valuable information not only on the mass and the interaction of DM but also on the lepton flavor structure [17]. Although the model shows interesting and promising features on phenomenology, the model cannot give any answer to the hierarchy problem unfortunately. Previous models are usually constructed in the nonsupersymmetric framework. Thus, the detailed study on the supersymmetrization of the model is one of the remaining subjects relevant to the model.

The supersymmetric extension of the model has been considered in [23, 24].4 Two DM candidates appear in these extensions as long as $R$-parity conservation is assumed. The existence of two kinds of $Z_{2}$ symmetry, that is, $R$-parity and $Z_{2}$ which forbids treelevel neutrino masses, guarantees the stability of these two DM candidates. In that case both of them contribute to the expected DM relic abundance 5 However, if one of them is unstable but has an longer life time than the age of universe, this decaying DM may cause the additional contribution cosmic ray, which brings anomalous excess of the charged particle flux over the expected background. Such a possibility has been studied in a supersymmetrized model in [24], where $Z_{2}$ is assumed to be weakly broken by the anomaly effect. Since the anomaly induced interaction is strongly suppressed, it causes

\footnotetext{
${ }^{3}$ There are a lot of study for the DM nature in radiative neutrino mass generation models and other phenomenological features of such models [5, 6, 7, 8, 9, 10, 11].

${ }^{4} \mathrm{~A}$ relevant supersymmetric model is considered in a different context in [25].

${ }^{5}$ Multi-component DM models have been considered in several contexts [26].
} 
the DM decay but its life time can be long enough.

In this paper, we consider a modification of the original one given in [24] by introducing the an anomalous U(1) gauge symmetry. Spontaneous breaking of this symmetry causes the weakly broken $Z_{2}$ symmetry at low energy regions naturally in addition to the conserved $R$-parity. It makes us possible to understand the smallness of some couplings through the Froggatt-Nielsen mechanism [27, 28], which is required to generate the small neutrino masses 6 Simultaneously, hierarchical masses of quarks and charged leptons are also explained by the same origin. The model also shows another interesting features relevant to the DM phenomenology. We give its analysis which includes the cosmic ray anomaly expected in this model, the characteristic gamma predicted in the DM radiative decay and the direct search of the stable DM through the elastic scattering with nuclei.

The paper is organized as follows. In section 2 we give the low energy effective model with an anomalous U(1) gauge symmetry. Spontaneous breaking of this symmetry gives the $Z_{2}$ symmetry which is relevant to the neutrino mass generation. The neutrino mass and mixing, the lepton flavor violating processes and the DM relic abundance are studied in this framework. In section 3 the nature of DM is discussed from a view point of both direct and indirect searches. Section 4 is devoted to the summary.

\section{A supersymmetric extension}

The radiative seesaw model proposed in [4] is an extension of the SM with three righthanded neutrinos and an inert doublet scalar.7 It has no coupling with quarks and no vacuum expectation value (VEV). Although the model has interesting phenomenological features as discussed in [5, 6, 8], it can not give an answer to the hierarchy problem. Thus, it is worthy to consider the supersymmetric extension of the model and to study phenomenology associated to it. The supersymmetrization of the model requires to introduce inert doublet chiral superfields $\eta_{u}$ and $\eta_{d}$ and also a singlet chiral superfield $\phi$ to the minimal supersymmetric SM (MSSM) [24]. They play the similar role as the inert doublet scalar in the original nonsupersymmetric model and bring required terms in the

\footnotetext{
${ }^{6}$ An anomalous $\mathrm{U}(1)$ symmetry has been considered in various phenomenological contexts [29]. In a radiative seesaw mechanism, it is also considered in [30. However, the present model is different from it in the nature of dark matter and also the $R$-parity conservation.

${ }^{7}$ The origin of the second doublet is discussed in 31 .
} 


\begin{tabular}{|c|c|c|c|c|c|c|}
\hline$\Psi$ & $Q_{i}$ & $\bar{D}_{i}$ & $L_{i}$ & $\bar{E}_{i}$ & $H_{u}$ & $H_{d}$ \\
\hline $\mathrm{SU}(2)_{L} \times \mathrm{U}(1)_{Y}$ & $\left(\underline{2}, \frac{1}{6}\right)$ & $\left(\underline{1}, \frac{-2}{3}\right)$ & $\left(\underline{2}, \frac{-1}{2}\right)$ & $(\underline{1}, 1)$ & $\left(\underline{2}, \frac{1}{2}\right)$ & $\left(\underline{2}, \frac{-1}{2}\right)$ \\
\hline$R$ & - & - & - & - & + & + \\
\hline$X$ & $2 n_{Q_{i}}$ & $2 n_{U_{i}}$ & $2 n_{L_{i}}$ & $2 n_{E_{i}}$ & $2 n_{H_{u}}$ & $2 n_{H_{d}}$ \\
\hline$Z_{2}$ & + & + & + & + & + & + \\
\hline$\Psi$ & $\bar{N}_{i}$ & $\eta_{u}$ & $\eta_{d}$ & $\phi$ & $\Sigma_{+}$ & $\overline{\Sigma_{-}}$ \\
\hline $\mathrm{SU}(2)_{L} \times \mathrm{U}(1)_{Y}$ & $(\underline{1}, 0)$ & $\left(\underline{2}, \frac{1}{2}\right)$ & $\left(\underline{1}, \frac{-1}{2}\right)$ & $(\underline{1}, 0)$ & $(\underline{1}, 0)$ & $(\underline{1}, 0)$ \\
\hline$R$ & + & - & - & - & + & + \\
\hline$X$ & $2 n_{N_{i}}+1$ & $2 n_{\eta_{u}}+1$ & $2 n_{\eta_{d}}+1$ & $2 n_{\phi}+1$ & $2 n_{+}$ & -2 \\
\hline$Z_{2}$ & - & - & - & - & + & + \\
\hline
\end{tabular}

Table 1: Matter contents and their quantum number. $X$ represents the charge of the anomalous $\mathrm{U}(1)_{X}$ and each $n_{\Psi}$ is an integer. $Z_{2}$ is a remnant symmetry of $\mathrm{U}(1)_{X}$ caused by the symmetry breaking due to $\left\langle\Sigma_{ \pm}\right\rangle \neq 0$.

Lagrangian for the neutrino mass generation. As the origin of the $Z_{2}$ symmetry which forbids tree level neutrino masses, we suppose the existence of an anomalous $\mathrm{U}(1)_{X}$ gauge symmetry. We assume that the $R$-parity is conserved. Matter contents of the model and their relevant quantum numbers are summarized in Table 1.

\subsection{A low energy effective model}

First, we discuss spontaneous breaking of the anomalous $U(1)_{X}$ symmetry at a high energy scale, which brings the low energy effective theory. Hierarchical couplings and masses are found to be generated in the low energy theory through this breaking. The vacuum is expected to be determined as the flat direction of the $D$-term of $\mathrm{U}(1)_{X}$. The $D$-term for relevant hidden fields is given by

$$
V_{D}=\frac{g_{X}^{2}}{2}\left[\left(2 n_{\phi}+1\right)|\phi|^{2}+2 n_{+}\left|\Sigma_{+}\right|^{2}-2\left|\Sigma_{-}\right|^{2}+\xi_{X}\right]^{2}
$$

$\xi_{X}$ is the $\mathrm{U}(1)_{X}$ Fayet-Iliopoulos $D$-term. It is expressed in string models as 32 .

$$
\xi_{X}=\frac{\operatorname{Tr} X}{192 \pi^{2}} g_{X}^{2} M_{\mathrm{pl}}^{2} \equiv \delta_{\mathrm{GS}} g_{X}^{2} M_{\mathrm{pl}}^{2},
$$

where $M_{\mathrm{pl}}$ is the reduced Planck mass and $X$ stands for the anomalous $\mathrm{U}(1)_{X}$ charge of the fields. On the other hand, the lowest order superpotential for these fields can be 
written as

$$
W_{h}=\frac{c_{+}}{M_{\mathrm{pl}}^{n_{+}-2}} \Sigma_{+} \Sigma_{-}^{n_{+}}+\frac{c_{\phi}}{M_{\mathrm{pl}}^{2 n_{\phi}}} \phi^{2} \Sigma_{-}^{2 n_{\phi}+1},
$$

where $c_{+}$and $c_{\phi}$ are considered as real constants. If we suppose supersymmetry breaking in the hidden sector, supersymmetry breaking terms appear in the scalar potential of the hidden sector. The scalar potential derived from $W_{h}$ may be written as

$$
\begin{aligned}
V_{F}= & \frac{c_{+}^{2}}{M_{\mathrm{pl}}^{2\left(n_{+}-2\right)}}\left|\Sigma_{-}\right|^{2 n_{+}}+\frac{4 c_{\phi}^{2}}{M_{\mathrm{pl}}^{4 n_{\phi}}}\left|\phi \Sigma_{-}^{2 n_{\phi}+1}\right|^{2}+\frac{c_{+}^{2} n_{+}^{2}}{M_{\mathrm{pl}}^{2\left(n_{+}-2\right)}}\left|\Sigma_{+} \Sigma_{-}^{n_{+}-1}\right|^{2} \\
& +\frac{c_{\phi}^{2}\left(2 n_{\phi}+1\right)^{2}}{M_{\mathrm{pl}}^{4 n_{\phi}}}\left|\phi^{2} \Sigma_{-}^{2 n_{\phi}}\right|^{2}+\left(\frac{c_{+} n_{+} c_{\phi}\left(2 n_{\phi}+1\right)}{M_{\mathrm{pl}}^{n_{+}+2 n_{\phi}-2}}\left(\Sigma_{+} \Sigma_{-}^{n_{+}-1}\right)^{*} \phi^{2} \Sigma_{-}^{2 n_{\phi}}+\text { h.c. }\right), \\
& -F_{1} \Sigma_{-}^{2}-F_{2} \Sigma_{+}^{2},
\end{aligned}
$$

where $F_{1}$ and $F_{2}$ represent the VEVs of $F$-components of some chiral superfields in the hidden sector. They bring the supersymmetry breaking of $O\left(10^{11}\right) \mathrm{GeV}$ in the hidden sector and also induce the soft terms of $O(1) \mathrm{TeV}$ through the gravity mediation in the observable sector.

Here we assume that the VEVs of $\Sigma_{ \pm}$and $\phi$ are real, for simplicity. Then, the minimization of the potential $V=V_{D}+V_{F}$ along the $D$-flat direction suggests that there exists a vacuum defined by

$$
\left\langle\Sigma_{-}\right\rangle \simeq \sqrt{\frac{\xi_{X}}{2}} \gg\left\langle\Sigma_{+}\right\rangle, \quad\langle\phi\rangle=0,
$$

as long as $c_{+}$is sufficiently suppressed. For example, if $\operatorname{Tr} X \sim 150 / g_{X}^{2}$ is satisfied, $\left\langle\Sigma_{-}\right\rangle \sim 0.2 M_{\mathrm{pl}}$ is expected. Moreover, $\left\langle\Sigma_{+}\right\rangle \simeq 10^{-4} M_{\mathrm{pl}}$ is also expected for sufficiently suppressed values of $c_{+}$such as $O\left(10^{-7}\right)$. Although this kind of vacuum can be realized only for the finely tuned parameters, we assume it in the following study.

If we adopt this vacuum to fix the low energy effective theory, the superpotential invariant under the imposed symmetry is obtained with the effectively induced parameters such as

$$
\begin{aligned}
W & =h_{i j}^{U} Q_{i} \bar{U}_{j} H_{u}+h_{i j}^{D} Q_{i} \bar{D}_{j} H_{d}+h_{i}^{E} L_{i} \bar{E}_{i} H_{d}+\mu_{H} H_{u} H_{d}, \\
& +h_{i j}^{N} L_{i} \bar{N}_{j} \eta_{u}+\lambda_{u} \eta_{u} H_{d} \phi+\lambda_{d} \eta_{d} H_{u} \phi+\mu_{\eta} \eta_{u} \eta_{d}+\frac{1}{2} M_{i} \bar{N}_{i} \bar{N}_{i}+\frac{1}{2} \mu_{\phi} \phi^{2} .
\end{aligned}
$$

The invariance of each term under $R \times \mathrm{U}(1)_{X}$ is guaranteed since the effective couplings and masses are generated through the $\operatorname{VEVs}\left\langle\Sigma_{ \pm}\right\rangle$as shown below (see also Appendix 
A). The MSSM superpotential is contained in the first line, while the second line includes additional terms to the MSSM. Yukawa couplings for the charged leptons and the mass matrix for the right-handed neutrinos are supposed to be flavor diagonal 8

The effective parameters in this superpotential are induced from higher order invariant interaction terms which contain a suitable number of $\Sigma_{ \pm}$. The dominant contribution are determined by the lowest order term of the following form:

$$
\begin{aligned}
& h_{i j k}=y_{i j k}\left(\frac{\left\langle\Sigma_{ \pm}\right\rangle}{M_{\mathrm{pl}}}\right)^{n_{i j k}}, \quad n_{i j k}=-\frac{X_{i}+X_{j}+X_{k}}{X_{\Sigma_{ \pm}}} \text {for } h_{i j k} \Psi_{i} \Psi_{j} \Psi_{k}, \\
& \mu_{i j}=y_{i j} M_{\mathrm{pl}}\left(\frac{\left\langle\Sigma_{ \pm}\right\rangle}{M_{\mathrm{pl}}}\right)^{n_{i j}}, \quad n_{i j}=-\frac{X_{i}+X_{j}}{X_{\Sigma_{ \pm}}} \text {for } \mu_{i j} \Psi_{i} \Psi_{j} .
\end{aligned}
$$

The original coupling constants $y_{i j k}$ and $y_{i j}$ in the nonrenormalizable interaction terms are considered to be values of $O(1)$. If the singlet scalars $\Sigma_{ \pm}$obtain the VEVs as discussed above, these VEVs cause hierarchical structure in the Yukawa couplings of quarks and leptons, and also suppress several parameters in the superpotential as found from eqs. (5) and (7). In fact, as long as suitable $\mathrm{U}(1)_{X}$ charges are assigned to quarks and leptons, we find that the hierarchical mass eigenvalues and mixing are generated via Froggatt-Nielsen mechanism [27]. In the similar way, the Yukawa couplings $\lambda_{u, d}$ are largely suppressed, and also $\mu_{\eta}$ and $M_{i}$ can take values of $O(1) \mathrm{TeV}$. These parameters are relevant to the neutrino mass generation and their values can be favorable for it as seen in the next part. We give more detailed discussion by giving such concrete examples for the charge assignment in the Appendix A. We also find that $\left\langle\Sigma_{ \pm}\right\rangle$breaks the anomalous $\mathrm{U}(1)_{X}$ symmetry spontaneously to its $Z_{2}$ subgroup. This is obvious from the charge assignment shown in Table 1 . This $Z_{2}$ symmetry plays a crucial role in the DM phenomenology.

Here we also note an interesting point related to the anomaly induced interaction. We have $L_{i} \eta_{u}$ as an only renormalizable operator which breaks the $\mathrm{U}(1)_{X}$ invariance but is invariant under the SM gauge symmetry and the $R$-parity. It is not included in the low energy superpotential (6) since we can not make it $\mathrm{U}(1)_{X}$ invariant by multiplying any number of $\Sigma_{ \pm}$. However, it can be $\mathrm{U}(1)_{X}$ invariant if it is accompanied by a dilaton chiral superfield $S$. As is well known, if the anomalous $\mathrm{U}(1)_{X}$ gauge transformation $V_{X} \rightarrow V_{X}+i\left(\Lambda-\Lambda^{\dagger}\right) / 2$ is associated with the shift of the dilaton field such as

$$
S \rightarrow S+i \delta_{\mathrm{GS}} \Lambda
$$

\footnotetext{
${ }^{8}$ This can be justified as long as the relevant original couplings $y_{i j k}$ and $y_{i j}$ in eq. (7) are flavor diagonal.
} 
the anomaly cancellation for $\mathrm{U}(1)_{X}$ is completed [33, 34]. Noting this fact, we observe that the following non-perturbative superpotential is also invariant under the imposed symmetry 34$] 9$

$$
\begin{aligned}
& W_{\mathrm{np}}=c_{i} M_{\mathrm{pl}} e^{-b_{i}} L_{i} \eta_{u}, \\
& b_{i}=\frac{\left(2 n_{i}^{L}+2 n_{\eta_{u}}+1\right)}{\delta_{\mathrm{GS}}} S \sim \frac{192 \pi^{2}\left(2 n_{i}^{L}+2 n_{\eta_{u}}+1\right)}{\operatorname{TrX}}
\end{aligned}
$$

where we use $\langle S\rangle$ and $c_{i}$ are constants of $O(1)$. The expression for $b_{i}$ shows that it is determined by the $\mathrm{U}(1)_{X}$ charge of the field contents of the model including the hidden sector. We also note that this term breaks the remnant $Z_{2}$ symmetry very weakly. The scale of its violation is determined by both the value of $\operatorname{Tr} X$ and the charges of $L_{i}$ and $\eta_{u}$.

Soft supersymmetry breaking terms associated with the superpotential $W$ and $W_{\mathrm{np}}$ are introduced as follows,

$$
\begin{aligned}
\mathcal{L}_{S B}= & -\tilde{m}_{\eta_{u}}^{2} \tilde{\eta}_{u}^{\dagger} \tilde{\eta}_{u}-\tilde{m}_{\eta_{d}}^{2} \tilde{\eta}_{d}^{\dagger} \tilde{\eta}_{d}-\tilde{m}_{\bar{N}}^{2} \tilde{\bar{N}}_{i}^{\dagger} \tilde{\bar{N}}_{i}-\tilde{m}_{\phi}^{2} \tilde{\phi}^{\dagger} \tilde{\phi} \\
& +A\left(h_{i j}^{N} \tilde{L}_{i} \tilde{\bar{N}}_{j} \tilde{\eta}_{u}+\lambda_{u} \tilde{\eta}_{u} H_{d} \tilde{\phi}+\lambda_{d} \tilde{\eta}_{d} H_{u} \tilde{\phi}+\text { h.c. }\right) \\
& -B\left(\mu_{H} H_{u} H_{d}+\mu_{\eta} \tilde{\eta}_{u} \tilde{\eta}_{d}+\frac{1}{2} \mu_{\phi} \tilde{\phi}^{2}+\frac{1}{2} M_{i} \tilde{\bar{N}}_{i}^{2}+c_{i} M_{\mathrm{pl}} e^{-b_{i}} \tilde{L}_{i} \tilde{\eta}_{u}+\text { h.c. }\right),
\end{aligned}
$$

where the additional part to the MSSM is listed alone. The scalar component is represented by putting a tilde on the character of the corresponding chiral superfield except for the ordinary Higgs chiral superfields $H_{u}$ and $H_{d}$. Universality of the soft supersymmetry breaking $A$ - and $B$-parameters is assumed to be satisfied here. Moreover, we confine our consideration to the case in which the soft scalar masses for all scalar partners of quarks and leptons are flavor diagonal and universal. They are denoted by $m_{0}$ in the following analysis and we assume $A=B=m_{0}$, for simplicity.

We should remind the reader that the soft scalar masses have the contributions from the anomalous $\mathrm{U}(1)_{X} D$-term such as

$$
\Delta \tilde{m}_{i}^{2}=g_{X}^{2} X_{i}\left(C_{h}+2 n_{H_{u}}\left\langle H_{u}^{0}\right\rangle^{2}+2 n_{H_{d}}\left\langle H_{d}^{0}\right\rangle^{2}\right)
$$

where $C_{h}$ stands for the remnant contribution from the hidden sector. Although these are flavor dependent, we confine our study to the case $m_{0}^{2} \gg \Delta \tilde{m}_{i}^{2}$, which may be realized on

\footnotetext{
${ }^{9}$ The effect of the appearance of this term has been studied in the case of discrete symmetry in [24].
} 
the vacuum with a finely tuned $C_{h}$. In such a case there is no new dangerous origin for the flavor changing neutral current (FCNC) caused by the soft supersymmetry breaking terms. In the following study the effective parameters in eq. (6) and also the soft supersymmetry breaking parameters in eq. (10) are treated as real except for $h_{i j}^{N}$, for simplicity.

\section{$2.2 \quad$ Neutrino mass and mixing}

Neutrino masses are generated through the one-loop diagram shown in Fig. 1 when the Higgs doublet scalars $H_{u}^{0}$ and $H_{d}^{0}$ obtain the VEVs. As found from eq. (40), since $\lambda_{u}$ and $\lambda_{d}$ are very small, the mixing between $\left(\tilde{\eta}_{u}^{0 *}, \tilde{\eta}_{d}^{0}\right)$ and $\left(\tilde{\phi}^{*}, \tilde{\phi}\right)$ can be treated as an insertion in the calculation of these diagrams with good accuracy. The mass matrices for $\left(\tilde{\eta}_{u}^{0 *}, \tilde{\eta}_{d}^{0}\right)$ and $\left(\tilde{\phi}^{*}, \tilde{\phi}\right)$ are written as

$$
\mathcal{M}_{\eta^{0}}=\left(\begin{array}{cc}
\bar{m}_{\eta_{u}}^{2} & B \mu_{\eta} \\
B \mu_{\eta} & \bar{m}_{\eta_{d}}^{2}
\end{array}\right), \quad \mathcal{M}_{\phi}=\frac{1}{2}\left(\begin{array}{cc}
\bar{m}_{\phi}^{2} & B \mu_{\phi} \\
B \mu_{\phi} & \bar{m}_{\phi}^{2}
\end{array}\right)
$$

where $\bar{m}_{\eta_{u, d}}^{2} \simeq m_{0}^{2}+\mu_{\eta}^{2}+\lambda_{u, d}^{2} v_{d, u}^{2}$ and $\bar{m}_{\phi}^{2} \simeq m_{0}^{2}+\mu_{\phi}^{2}+\lambda_{u}^{2} v_{d}^{2}+\lambda_{d}^{2} v_{u}^{2}$. If we define the mass eigenstates of these mass matrices by

$$
\left(\begin{array}{c}
\tilde{\eta}_{+} \\
\tilde{\eta}_{-}
\end{array}\right)=\left(\begin{array}{cc}
\cos \theta_{\eta} & \sin \theta_{\eta} \\
-\sin \theta_{\eta} & \cos \theta_{\eta}
\end{array}\right)\left(\begin{array}{c}
\tilde{\eta}_{u}^{0 *} \\
\tilde{\eta}_{d}^{0}
\end{array}\right), \quad\left(\begin{array}{c}
\tilde{\phi}_{+} \\
\tilde{\phi}_{-}
\end{array}\right)=\left(\begin{array}{cc}
\cos \theta_{\phi} & \sin \theta_{\phi} \\
-\sin \theta_{\phi} & \cos \theta_{\phi}
\end{array}\right)\left(\begin{array}{c}
\tilde{\phi}^{*} \\
\tilde{\phi}
\end{array}\right),
$$

the mass eigenvalues and the mixing angles can be written as

$$
\begin{aligned}
& m_{\eta \pm}^{2}=\frac{1}{2}\left(\bar{m}_{\eta_{u}}^{2}+\bar{m}_{\eta_{d}}^{2} \pm \sqrt{\left(\bar{m}_{\eta_{u}}^{2}-\bar{m}_{\eta_{d}}^{2}\right)^{2}+4 B^{2} \mu_{\eta}^{2}}\right), \quad \tan 2 \theta_{\eta}=\frac{2 B \mu_{\eta}}{\bar{m}_{\eta_{d}}^{2}-\bar{m}_{\eta_{u}}^{2}} \\
& m_{\phi \pm}^{2}=\bar{m}_{\phi}^{2} \pm B \mu_{\phi}, \quad \theta_{\phi}=\frac{\pi}{4} .
\end{aligned}
$$

We find that the scalar superpartners $\left(\tilde{\bar{N}}_{i}^{*}, \tilde{\bar{N}}_{i}\right)$ of $\bar{N}_{i}$ satisfy the same relations as the ones of $\left(\tilde{\phi}^{*}, \tilde{\phi}\right)$ as shown above. Their mass eigenvalues $M_{i \pm}^{2}$ can be read off from the expression for $m_{\phi \pm}^{2}$ by replacing $\mu_{\phi}$ and $\bar{m}_{\phi}^{2}$ with $M_{i}$ and $m_{0}^{2}+M_{i}^{2}$, respectively.

We can calculate the neutrino masses generated through the one-loop diagrams by using these. Since $\mu_{\phi}^{2}$ is expected to be larger than $\mu_{\eta}^{2}$ and $m_{0}^{2}$, we find that the dominant contribution is caused by the diagram (a) in Fig. 1 and it is estimated as

$$
\begin{aligned}
\left(\mathcal{M}_{\nu}\right)_{\alpha \beta}= & \frac{\lambda_{u} \lambda_{d} v_{u} v_{d} \sin 2 \theta_{\eta}}{16 \pi^{2}} \sum_{i=1}^{3} h_{\alpha i} h_{\beta i} M_{i}\left(g\left(M_{i}, m_{\eta+}\right) \cos ^{2} \theta_{\eta}-g\left(M_{i}, m_{\eta-}\right) \sin ^{2} \theta_{\eta}\right. \\
& \left.-f\left(M_{i}, m_{\eta+}, m_{\eta-}\right) \cos 2 \theta_{\eta}\right)
\end{aligned}
$$



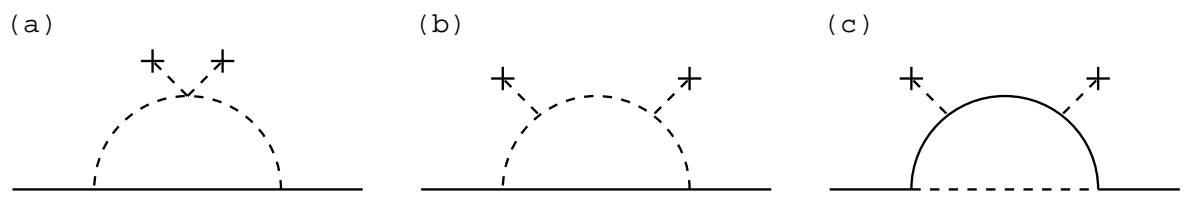

Fig. 1 One-loop diagrams contributing to the neutrino masses. Two diagrams (b) and (c) have an internal line of the scalar $\tilde{\phi}$ or its fermionic partner, respectively.

where $f$ and $g$ are defined as

$$
\begin{aligned}
& f\left(m_{a}, m_{b}, m_{c}\right)=\frac{m_{a}^{2} m_{b}^{2} \ln \left(m_{b}^{2} / m_{a}^{2}\right)+m_{b}^{2} m_{c}^{2} \ln \left(m_{c}^{2} / m_{b}^{2}\right)+m_{c}^{2} m_{a}^{2} \ln \left(m_{a}^{2} / m_{c}^{2}\right)}{\left(m_{c}^{2}-m_{a}^{2}\right)\left(m_{a}^{2}-m_{b}^{2}\right)\left(m_{b}^{2}-m_{c}^{2}\right)}, \\
& g\left(m_{a}, m_{b}\right)=\frac{m_{b}^{2}-m_{a}^{2}+m_{a}^{2} \ln \left(m_{a}^{2} / m_{b}^{2}\right)}{\left(m_{b}^{2}-m_{a}^{2}\right)^{2}} .
\end{aligned}
$$

Other two diagrams (b) and (c) with the component of $\phi$ as an internal line are expected to bring the subdominant contributions. We give the explicit expressions of their contributions in the Appendix B. The universal soft supersymmetry breakings make the situation simple since $\theta_{\eta}=\pi / 4$ is satisfied. If $M_{i}$ and $\bar{m}_{\eta_{u, d}}$ have the values of $O(1) \mathrm{TeV}$, the mass eigenvalues of neutrinos must be controlled by a small parameter $\lambda_{u} \lambda_{d}$ of $O\left(10^{-8}\right)$. As long as the anomalous $\mathrm{U}(1)_{X}$ charge is assigned suitably, these can be naturally realized as found from the examples given in the Appendix A.

Here we focus our attention to the flavor structure of the neutrino Yukawa coupling:10 [6. 7]

$$
h_{e i}^{N}=0, \quad h_{\mu i}^{N}=h_{\tau i}^{N} \equiv h_{i}(i=1,2), \quad h_{e 3}^{N}=h_{\mu 3}^{N}=-h_{\tau 3}^{N} \equiv h_{3} .
$$

This flavor structure is consistent with the $\mathrm{U}(1)_{X}$ invariance as long as all of $y_{i j}^{N}$ take the same values except for $y_{e i}^{N}$, which should be assumed to be $y_{e i}^{N}=0$ for $i=1,2$ (see Appendix A). An interesting point of this flavor structure is that the neutrino mass matrix in eq.(15) takes the following simple form:

$$
\mathcal{M}_{\nu}=\left(\begin{array}{lll}
0 & 0 & 0 \\
0 & 1 & 1 \\
0 & 1 & 1
\end{array}\right)\left(h_{1}^{2} \Lambda_{1}+h_{2}^{2} \Lambda_{2}\right)+\left(\begin{array}{ccc}
1 & 1 & -1 \\
1 & 1 & -1 \\
-1 & -1 & 1
\end{array}\right) h_{3}^{2} \Lambda_{3}
$$

\footnotetext{
${ }^{10}$ This structure is chosen adhoc here since it has several interesting features as shown below.
} 
where $\Lambda_{i}$ fixes the mass scale for the neutrino masses as follows, 11

$$
\Lambda_{i}=\frac{\bar{\lambda} v^{2} M_{i}}{32 \pi^{2}}\left(g\left(M_{i}, m_{\eta+}\right)-g\left(M_{i}, m_{\eta_{-}}\right)\right), \quad \bar{\lambda} \equiv \frac{\lambda_{u} \lambda_{d} \tan \beta}{1+\tan ^{2} \beta},
$$

where $\sin \beta=v_{u} / v$ and $\cos \beta=v_{d} / v$. This mass matrix automatically derives the tribimaximal mixing, which is favored by the neutrino oscillation data. In fact, it is easily checked that the MNS matrix for this neutrino mass model is given by

$$
U_{M N S}=\left(\begin{array}{ccc}
\frac{2}{\sqrt{6}} & \frac{1}{\sqrt{3}} & 0 \\
\frac{-1}{\sqrt{6}} & \frac{1}{\sqrt{3}} & \frac{1}{\sqrt{2}} \\
\frac{1}{\sqrt{6}} & \frac{-1}{\sqrt{3}} & \frac{1}{\sqrt{2}}
\end{array}\right)\left(\begin{array}{ccc}
1 & 0 & 0 \\
0 & e^{i \alpha_{1}} & 0 \\
0 & 0 & e^{i \alpha_{2}}
\end{array}\right)
$$

where Majorana phases $\alpha_{1,2}$ are expressed as

$$
\alpha_{1}=\varphi_{3}, \quad \alpha_{2}=\frac{1}{2} \tan ^{-1}\left(\frac{\left|h_{1}\right|^{2} \Lambda_{1} \sin 2 \varphi_{1}+\left|h_{2}\right|^{2} \Lambda_{2} \sin 2 \varphi_{2}}{\left|h_{1}\right|^{2} \Lambda_{1} \cos 2 \varphi_{1}+\left|h_{2}\right|^{2} \Lambda_{2} \cos 2 \varphi_{2}}\right)
$$

by using $\varphi_{i}=\arg \left(h_{i}\right)$. Here it should be reminded that Majorana phases $\alpha_{1,2}$ do not affect the neutrino oscillations.

Taking account of the nature discussed above and also the fact that one of the eigenvalues of the mass matrix (18) is zero, the remaining mass eigenvalues are found to be equal to $\sqrt{\Delta m_{\text {atm }}^{2}}$ and $\sqrt{\Delta m_{\text {sol }}^{2}}$. This is required for the explanation of neutrino oscillation data. Thus, we find that the model parameters should satisfy the relations

$$
\left|h_{1}^{2} \Lambda_{1}+h_{2}^{2} \Lambda_{2}\right| \simeq \frac{\sqrt{\Delta m_{\mathrm{atm}}^{2}}}{2}, \quad\left|h_{3}^{2} \Lambda_{3}\right| \simeq \frac{\sqrt{\Delta m_{\mathrm{sol}}^{2}}}{3} .
$$

The neutrino Yukawa couplings $h_{i}$ and the right-handed neutrino masses $M_{i}$ should correlate each other so as to satisfy these relations. Phenomenological study of the model should be proceeded under these constraints. In the following discussion, we restrict our study to the case with $M_{1} \lesssim M_{2}<M_{3}$, which allows us to take $\Lambda_{1} \simeq \Lambda_{2} 12$ Thus, free parameters relevant to the analysis of DM phenomenology are summarized as

$$
M_{1}, \quad M_{3}, \quad \bar{\lambda}, \quad \mu_{\eta}, \quad m_{0} .
$$

\footnotetext{
${ }^{11}$ If the mass matrix is modified from eq. (15) due to other contributions neglected as the subdominant ones here, the ambiguity is confined into the $\Lambda_{i}$. The MNS matrix is not affected as long as the condition (17) is satisfied.

${ }^{12}$ It may be useful to note that this case has some advantages as discussed in [7, 17, but not only for simplicity.
} 

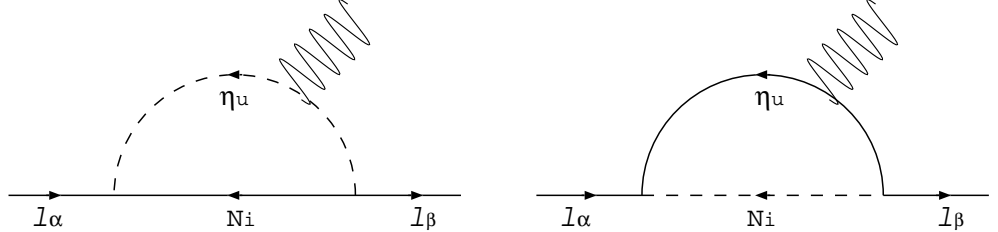

Fig. 2 One-loop diagrams causing the lepton flavor violating processes $\ell_{\alpha} \rightarrow \ell_{\beta} \gamma$.

If we suppose fixed the values for $M_{i}, \mu_{\eta}$ and $\tilde{m}_{0}$, the conditions (22) determine a value of $\bar{\lambda} \sqrt{\left|h_{1}^{2}+h_{2}^{2}\right|}$ and $\bar{\lambda}\left|h_{3}\right|$. A larger $\bar{\lambda}$ gives a smaller value of neutrino Yukawa couplings $\bar{\lambda} \sqrt{\left|h_{1}^{2}+h_{2}^{2}\right|}$ and $\bar{\lambda}\left|h_{3}\right|$. This feature becomes crucial when the constraints from lepton flavor violating processes and DM relic abundance are taken into account.

\subsection{Constraints from lepton flavor violating processes}

Since we suppose the slepton mass matrix is flavor diagonal and universal, there are no new FCNC source in the slepton sector. The FCNC is induced only through the MNS matrix elements which appear in the Higgsino exchange diagrams. Thus, if Higgsinos are much heavier than gauginos, the ordinary contributions to the FCNC caused by the supersymmetric partners are sufficiently suppressed in this model. On the other hand, the extension for the neutrino mass generation induces the one-loop contribution to the lepton flavor violating processes such as $\mu \rightarrow e \gamma$ as in the nonsupersymmetric case. Their diagrams are shown in Fig. 2. Since these diagrams do not need the mixing between $\eta_{u}$ and $\eta_{d}$ unlike the case of neutrino masses (see Fig. 1), it causes the large contributions to these processes. In that case the dominant contributions to the lepton flavor violating processes are given by these diagrams. They give the constraints on the model, which is the different from the ones in the MSSM. These processes may be used as the probe of the model in the future experiments.

Branching ratio of the lepton flavor violating process $\ell_{\alpha} \rightarrow \ell_{\beta} \gamma$ is given as

$$
\begin{aligned}
\operatorname{Br}\left(\ell_{\alpha}^{-} \rightarrow \ell_{\beta}^{-} \gamma\right)= & \frac{3 \alpha}{64 \pi}\left|\sum_{i=1}^{3} h_{\alpha i} h_{\beta i}^{*} \sum_{a= \pm}\left[\frac{1}{2 G_{F} m_{\eta a}^{2}} F_{2}\left(\frac{M_{i}^{2}}{m_{\eta a}^{2}}\right)+\frac{a}{2 G_{F} \mu_{\eta}^{2}} F_{2}\left(\frac{M_{i a}^{2}}{\mu_{\eta}^{2}}\right)\right]\right|^{2} \\
& \times B r\left(\ell_{\alpha}^{-} \rightarrow \ell_{\beta}^{-} \bar{\nu}_{\beta} \nu_{\alpha}\right)
\end{aligned}
$$

where $m_{\eta a}$ and $M_{i a}$ are the mass eigenvalues defined in eq. (14) and the statements below 

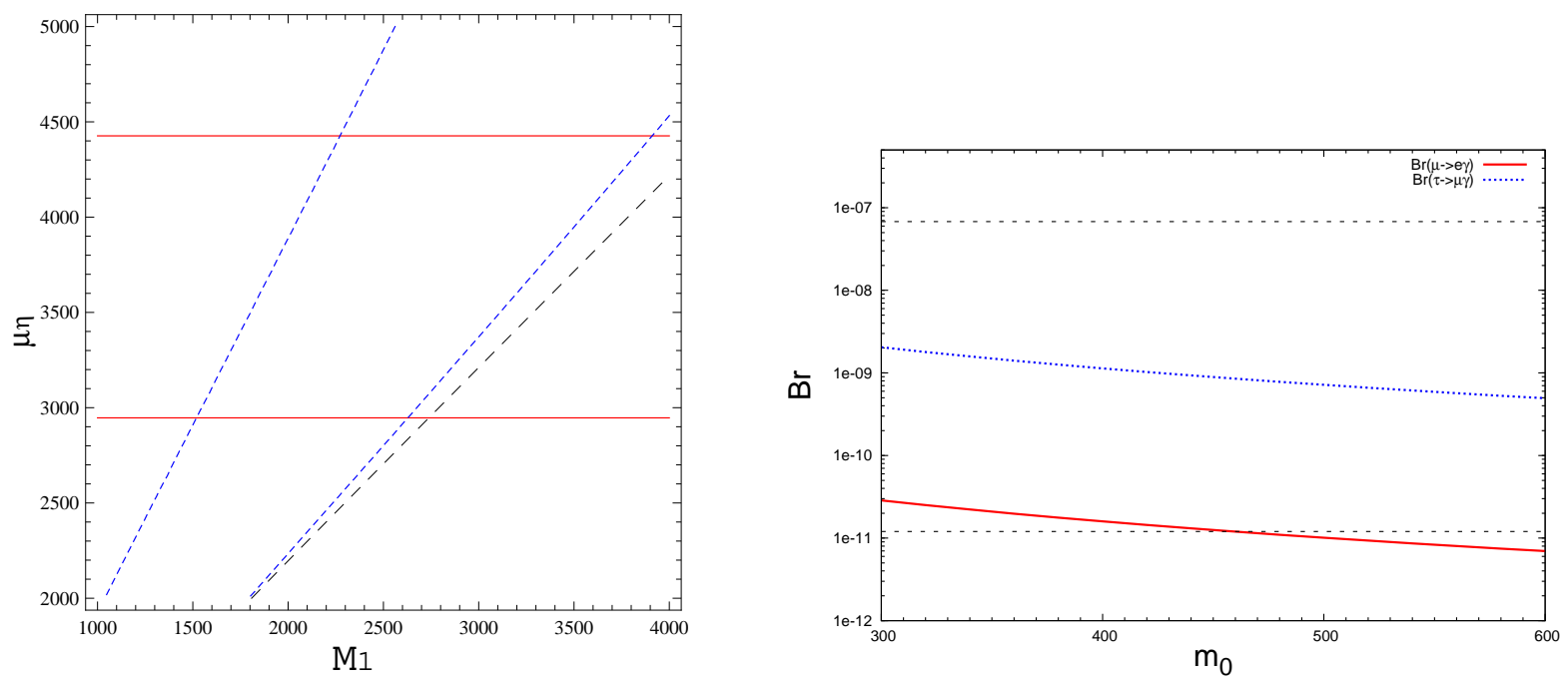

Fig. 3 The left frame shows the contours for $\operatorname{Br}(\mu \rightarrow e \gamma)$ (red solid lines) and $\operatorname{Br}(\tau \rightarrow \mu \gamma)$ (blue dashed lines) under the conditions (22) imposed by the neutrino oscillation data. A thin black dashed line represents a line for $m_{\eta-}=M_{1}$. The right frame shows the $m_{0}$ dependence of each branching ratio. Thin black dotted lines represent the present experimental bounds for $\mu \rightarrow e \gamma$ and $\tau \rightarrow \mu \gamma$, respectively. We use a $\mathrm{GeV}$ unit for $M_{1}, m_{0}$ and $\mu_{\eta}$.

it respectively. The function $F_{2}(x)$ is defined as

$$
F_{2}(x)=\frac{1-6 x+3 x^{2}+2 x^{3}-6 x^{2} \ln x}{6(1-x)^{4}} .
$$

If we use the assumed flavor structure for the neutrino Yukawa couplings (17), we find that

$$
\begin{aligned}
B r(\mu \rightarrow e \gamma) \simeq & \frac{3 \alpha\left|h_{3}\right|^{4}}{64 \pi}\left[\sum_{a= \pm}\left\{\frac{1}{2 G_{F} m_{\eta a}^{2}} F_{2}\left(\frac{M_{3}^{2}}{m_{\eta a}^{2}}\right)+\frac{a}{2 G_{F} \mu_{\eta}^{2}} F_{2}\left(\frac{M_{3 a}^{2}}{\mu_{\eta}^{2}}\right)\right\}\right]^{2} \\
B r(\tau \rightarrow \mu \gamma) \simeq & \frac{0.51 \alpha}{64 \pi}\left[\sum _ { a = \pm } \left\{\frac{1}{2 G_{F} m_{\eta a}^{2}}\left(\left(\left|h_{1}\right|^{2}+\left|h_{2}\right|^{2}\right) F_{2}\left(\frac{M_{1}^{2}}{m_{\eta a}^{2}}\right)-\left|h_{3}\right|^{2} F_{2}\left(\frac{M_{3}^{2}}{m_{\eta a}^{2}}\right)\right)\right.\right. \\
& \left.\left.+\frac{a}{2 G_{F} \mu_{\eta}^{2}}\left(\left(\left|h_{1}\right|^{2}+\left|h_{2}\right|^{2}\right) F_{2}\left(\frac{M_{1 a}^{2}}{\mu_{\eta}^{2}}\right)-\left|h_{3}\right|^{2} F_{2}\left(\frac{M_{3 a}^{2}}{\mu_{\eta}^{2}}\right)\right)\right\}\right]^{2}
\end{aligned}
$$

Now we examine the parameter regions consistent with both the neutrino oscillation data and the lepton flavor violating constraints. By using the above formulas for the lepton flavor violating processes and the neutrino oscillation condition (22), the branching ratio of $\mu \rightarrow e \gamma$ and $\tau \rightarrow \mu \gamma$ predicted by the model can be plotted in the $\left(M_{1}, \mu_{\eta}\right)$ plane if $M_{3}, m_{0}$ and $\bar{\lambda}$ are settled. It is useful to note that these bounds become more severe by 
making $\bar{\lambda}$ smaller generally since a smaller $\bar{\lambda}$ requires larger values for $\left|h_{i}\right|$ as found from eqs. (18) and (19). We fix these values to

$$
M_{3}=7.8 \mathrm{TeV}, \quad m_{0}=0.48 \mathrm{TeV}, \quad \bar{\lambda}=1.24 \times 10^{-9}
$$

as a typical example. The last one corresponds to $\lambda_{u} \lambda_{d}=10^{-7.7}$ and $\tan \beta=16$, for example. For a while, we consider the case $\varphi_{1}=\varphi_{2}$ only.

In the left frame of Fig. 3, we show the contours of these branching ratios. Red solid lines represent the contours of $\operatorname{Br}(\mu \rightarrow e \gamma) \times 10^{11}=1.2$ and 0.6 downward, and blue dotted ones are contours of $\operatorname{Br}(\tau \rightarrow \mu \gamma) \times 10^{8}=0.1$ and 0.04 rightward. The former one is independent of $M_{1}$ as is clear from the expression in eq. (26) $) . F_{2}\left(M_{3}^{2} / m_{\eta a}^{2}\right)$ becomes smaller for a larger $M_{3}$ although the larger $M_{3}$ makes the neutrino Yukawa coupling $h_{3}$ larger through eq.(22). Thus, this branching ratio can easily satisfy the present experimental bounds by making $M_{3}$ large enough. We note that this feature is intimately related to the flavor structure (17) which induces the tri-bimaximal MNS matrix. $\operatorname{Br}(\tau \rightarrow \mu \gamma)$ is found to show a different behavior, which is mainly controlled by the masses and the couplings of $\bar{N}_{1,2}$. This figure shows that the present experimental bounds for these processes [35] are satisfied at the wide range of the $m_{\eta-}>M_{1}$ regions, where $m_{\eta-}=M_{1}$ is plotted by a thin black dashed line.

In the right frame of Fig. 3, as in the left frame each branching ratio is plotted by a red solid line and a blue dotted line as a function of $m_{0}$ for $M_{1}=2.5 \mathrm{TeV}$ and $\mu_{\eta}=4.2 \mathrm{TeV}$. The present bounds for them are also plotted by the thin black solid and dashed lines, respectively. The figure suggests that the constraint from the $\mu \rightarrow e \gamma$ requires $m_{0} \gtrsim 0.45 \mathrm{TeV}$, although the $\tau \rightarrow \mu \gamma$ gives no constraint on $m_{0}$. From these figures, we find that the model can be easily consistent with both the neutrino oscillation data and the lepton flavor violating constraints for the natural values of parameters as long as they are suitably fixed.

As a related subject, it is useful to note that the similar diagram to Fig. 2 contributes to the electric dipole moment of an electron (EDME) and the muon $g-2$. Even if the neutrino Yukawa couplings $h_{e i}^{N}$ are complex, the EDME is not induced since there is no mixing among $\bar{N}_{i}$ 's. New contributions to the muon $g-2$ due to the similar diagram to 
Fig. 2 are summarized as

$$
\begin{aligned}
\delta a_{\mu} \simeq \frac{m_{\mu}^{2}}{2(4 \pi)^{2}} \sum_{a= \pm} & {\left[\frac{-1}{m_{\eta a}^{2}}\left\{\left(\left|h_{1}\right|^{2}+\left|h_{2}\right|^{2}\right) F_{2}\left(\frac{M_{1}^{2}}{m_{\eta a}^{2}}\right)+\left|h_{3}\right|^{2} F_{2}\left(\frac{M_{3}^{2}}{m_{\eta a}^{2}}\right)\right\}\right.} \\
& \left.+\frac{a}{\mu_{\eta}^{2}}\left\{\left(\left|h_{1}\right|^{2}+\left|h_{2}\right|^{2}\right) F_{2}\left(\frac{M_{1 a}^{2}}{\mu_{\eta}^{2}}\right)+\left|h_{3}\right|^{2} F_{2}\left(\frac{M_{3 a}^{2}}{\mu_{\eta}^{2}}\right)\right\}\right] .
\end{aligned}
$$

We estimate it for the allowed parameter sets obtained in the above analysis. The results seem to be smaller by three order of magnitude in comparison with $\delta a_{\mu}=(30.2 \pm 8.7) \times$ $10^{-10}$, which is a discrepancy shown by the SM prediction and the value derived by the experiment [36]. This suggests that another origin is required for the explanation of this muon $g-2$ discrepancy.

\subsection{Two dark matter candidate}

The model has two types of the DM candidate in general. One of them is the lightest neutralino $\chi$ whose stability is guaranteed by the $R$-parity as in case of the MSSM. The other one is the lightest neutral field with the odd parity of the new $Z_{2}$ symmetry, which is the remnant symmetry of the anomalous $\mathrm{U}(1)_{X}$. It corresponds to the lightest neutral state composed of the components of the chiral supermultiplets $\bar{N}_{i}$, or $\eta_{u, d}^{0}$ and $\phi$. In the following study, we assume that the singlet fermion $\psi_{N_{1}}$ (the fermionic component of $\bar{N}_{1}$ ) is the lightest one among these candidates. Since this $Z_{2}$ is not an exact symmetry but is weakly broken as shown in eq. (9) by the anomaly effect, the latter candidate $\psi_{N_{1}}$ is not stable but it could have a long lifetime comparable to the age of the universe. The condition for this possibility is discussed in the next section. If this is the case, the DM relic abundance suggested by the WMAP [1] should be satisfied by these two contributions such as

$$
\Omega_{\chi} h^{2}+\Omega_{\psi_{N_{1}}} h^{2}=0.11
$$

The annihilation of two $\psi_{N_{1}}$ 's is induced through the $t$ - and $u$-channel $\eta_{u}$ exchange. Final states of such processes are composed of a pair of lepton and antilepton or a pair of slepton and antislepton. The latter final state gives new contributions compared with the non-supersymmetric case as long as $M_{1}>\tilde{m}_{L}\left(\equiv m_{0}\right)$ is satisfied for the slepton mass $\tilde{m}_{L}$. If $\psi_{N_{2}}$ has a mass almost degenerate with the one of $\psi_{N_{1}}$ and this is the case here, we need to consider the coannihilation effect [37]. Final states of this annihilation are controlled by the flavor structure of the neutrino Yukawa couplings which is shown in eq. (17). On 

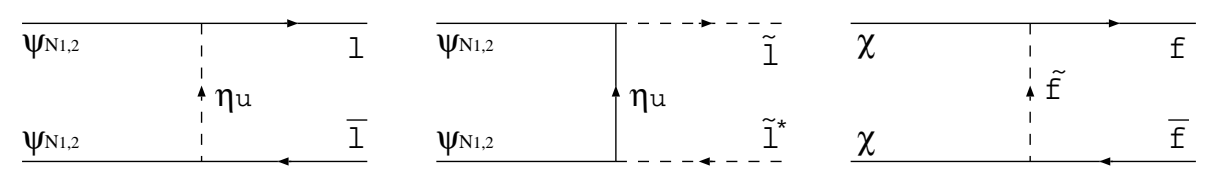

Fig. 4 Diagrams contributing to the $t$-channel annihilation of the two DM candidates. There are also $u$-channel diagrams corresponding to these. Although the final state $f \bar{f}$ of the $\chi$ annihilation is composed of both $q \bar{q}$ and $\ell \bar{\ell}$, the $\psi_{N_{1}}$ annihilation contains $\ell \bar{\ell}$ only.

the other hand, the annihilation of two $\chi$ 's occurs through various processes depending on its composition, which is determined by both the supersymmetry breaking scenario and the radiative symmetry breaking conditions (see Fig. 4). It has been studied in detail in the MSSM context [3]. Here we do not fix the scenario but just confine our study to the pure bino case by assuming that $\mu$ and the masses of Higgs doublet and gauginos are tuned to satisfy the required condition, for simplicity. In this case, the annihilation is expected to occur dominantly through $t$ - and $u$-channel sfermion exchange 13 We estimate the annihilation cross section $\sigma v$ for these DM candidates by expanding it as $\sigma v=a+b v^{2}$ in powers of their relative velocity $v$ [38].

For the singlet fermion $\psi_{N_{1}}$, we need to take account of the coannihilation effect with $\psi_{N_{2}}$ because of the assumption $M_{1} \simeq M_{2}$. In order to estimate the freeze-out temperature $T_{f}$ of $\psi_{N_{1}}$ including the coannihilation case, we follow the procedure given in [37]. We define $\sigma_{\text {eff }}$ and $g_{\text {eff }}$ as

$$
\begin{aligned}
\sigma_{\mathrm{eff}} & =\frac{g_{N_{1}}^{2}}{g_{\mathrm{eff}}^{2}} \sigma_{\psi_{N_{1}} \psi_{N_{1}}}+2 \frac{g_{N_{1}} g_{N_{2}}}{g_{\mathrm{eff}}^{2}} \sigma_{\psi_{N_{1}} \psi_{N_{2}}}(1+\delta)^{3 / 2} e^{-x \delta}+\frac{g_{N_{2}}^{2}}{g_{\mathrm{eff}}^{2}} \sigma_{\psi_{N_{2}} \psi_{N_{2}}}(1+\delta)^{3} e^{-2 x \delta}, \\
g_{\mathrm{eff}} & =g_{N_{1}}+g_{N_{2}}(1+\delta)^{3 / 2} e^{-x \delta},
\end{aligned}
$$

where internal degrees of freedom of $\bar{N}_{i}$ are described by $g_{N_{i}}$ and $\delta \equiv\left(M_{2}-M_{1}\right) / M_{1}$. If we define $a_{\text {eff }}$ and $b_{\text {eff }}$ by $\sigma_{\text {eff }} v=a_{\text {eff }}+b_{\text {eff }} v^{2}$, the thermally averaged cross section can be written as $\left\langle\sigma_{\text {eff }} v\right\rangle=a_{\text {eff }}+6 b_{\text {eff }} / x$ where $x=M_{1} / T$. Since $\delta \ll 1$ is supposed here, the second and third terms can bring the important contribution. Using these formulas, the

\footnotetext{
${ }^{13}$ If the lightest neutralino $\chi$ is heavier than Higgs scalars, we need to take account of the $t$-channel Higgsino exchange process. However, it is expected to be subdominant as long as the Higgsino is heavier than the sfermions, which is assumed throughout this analysis.
} 
effective annihilation cross section is given by

$$
\begin{aligned}
\left(\sigma_{\psi_{N_{1}}}\right)_{\mathrm{eff}} v & \simeq \frac{Y_{s}^{4}}{8 \pi} \sum_{a, b= \pm} \frac{M_{1}^{2}}{\left(M_{1}^{2}+m_{\eta a}^{2}\right)\left(M_{1}^{2}+m_{\eta b}^{2}\right)}\left(1+p_{F} v^{2}\right) \\
& +\frac{Y_{p}^{4}}{96 \pi} \sum_{a, b= \pm} \frac{M_{1}^{2}\left(M_{1}^{4}+m_{\eta a}^{2} m_{\eta b}^{2}\right)}{\left(M_{1}^{2}+m_{\eta a}^{2}\right)^{2}\left(M_{1}^{2}+m_{\eta b}^{2}\right)^{2}} v^{2} \\
& +\frac{Y_{s}^{4}}{8 \pi} \frac{M_{1}^{2} \beta}{\left(\mu_{\eta}^{2}+M_{1}^{2} \beta^{2}\right)^{2}}\left(\beta^{2}+p_{S} v^{2}\right) \\
& +\frac{Y_{p}^{4}}{32 \pi} \frac{M_{1}^{2} \beta}{\left(\mu_{\eta}^{2}+M_{1}^{2} \beta^{2}\right)^{2}}\left(1+\frac{2 \beta^{2}}{3}-\frac{4 \mu_{\eta}^{2} M_{1}^{2} \beta^{2}}{3\left(\mu_{\eta}^{2}+M_{1}^{2} \beta^{2}\right)^{2}}\right) v^{2}
\end{aligned}
$$

where $\beta=\sqrt{1-\left(m_{0} / M_{1}\right)^{2}}$. The mass eigenvalue $m_{\eta a}$ is given in eq. (14). $Y_{s}$ and $Y_{p}$ are defined by

$$
Y_{s}^{4}=2\left|h_{1}\right|^{2}\left|h_{2}\right|^{2} \sin ^{2}\left(\varphi_{1}-\varphi_{2}\right), \quad Y_{p}^{4}=\left|h_{1}\right|^{4}+2\left|h_{1}\right|^{2}\left|h_{2}\right|^{2} \cos 2\left(\varphi_{1}-\varphi_{2}\right)+\left|h_{2}\right|^{4}
$$

The first and second lines in eq. (31) represent the contributions with the lepton-antilepton final state. The third and forth lines come from the slepton-antislepton final state. We find that there can be $s$-wave contributions in the first and third lines if the neutrino Yukawa couplings $h_{i j}^{N}$ have the phases such as $\varphi_{1} \neq \varphi_{2}+n \pi 14$ This happens since the coannihilation cross section $\sigma_{\psi_{N_{1}} \psi_{N_{2}}}$ allows the $s$-wave contribution [7].

On the other hand, the $s$-wave contribution to the annihilation cross section of the bino-like $\chi$ to $\bar{f} f$ is expressed as 38 .

$$
\sigma_{\chi \chi} v \simeq \sum_{f} \frac{4 c_{f}}{\pi} \frac{G_{F}^{2} m_{\chi}^{2} m_{W}^{4} \beta^{\prime}}{\left(m_{0}^{2}+\beta^{\prime 2} m_{\chi}^{2}\right)^{2}} \tan ^{4} \theta_{W}\left(\left(T_{3 L}-Q_{f}\right)^{4}+Q_{f}^{4}+2\left(T_{3 L}-Q_{f}\right)^{2} Q_{f}^{2}\right) z^{2}
$$

where $z=m_{f} / m_{\chi}, \beta^{\prime}=\left(1-z^{2}\right)^{1 / 2}$ and $Q_{f}$ is the electric charge of $f$. In the summation in eq. (33), $f$ runs among quarks $\left(c_{f}=3\right)$ and leptons $\left(c_{f}=1\right)$ which satisfy $m_{f}<m_{\chi}$. Although $\chi \chi$ can annihilate to a pair of Higgs scalars through the Higgsino exchange, we neglect it as a subdominant process. If the bino mass $M_{\tilde{B}}$ is almost degenerate with Higgsino mass $\mu_{H}$, the coannihilation between $\chi$ and a Higgsino-like neutralino $\chi^{\prime}$ brings important effects on the relic abundance of $\chi$. The relevant cross sections to

\footnotetext{
${ }^{14}$ The $s$-wave contributions are dominant in such cases. The expressions for $p_{F, S}$ for the corresponding $p$-wave contributions are shown in Appendix C for the completeness.
} 

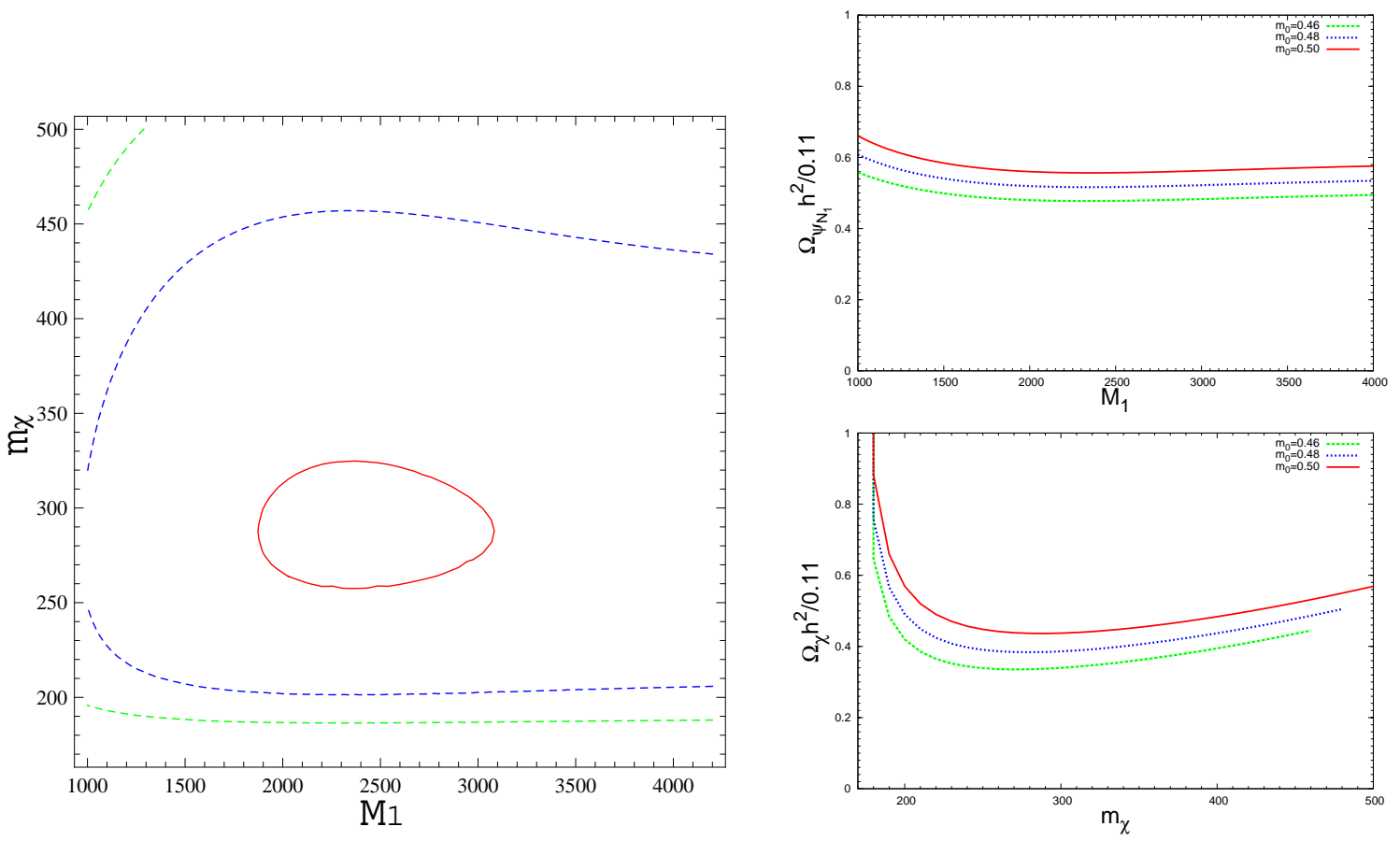

Fig. 5 The left frame shows the contours of $\Omega_{\psi_{N_{1}}} h^{2}+\Omega_{\chi} h^{2}=0.11$ for $m_{0}=0.46 \mathrm{TeV}$ (a green dotted line), $0.48 \mathrm{TeV}$ (a blue dotted line), and $0.50 \mathrm{TeV}$ (a red solid line). Each figure in the right frames shows the ratio of each DM component to the total relic abundance $\Omega_{\psi_{N_{1}}} h^{2} / 0.11$ and $\Omega_{\chi} h^{2} / 0.11$, respectively. Each line corresponds to the same value of $m_{0}$ used in the left frame. In these figures $\varphi_{1}-\varphi_{2}=0$ is assumed.

this coannihilation are given by [38]

$$
\begin{aligned}
\sigma_{\chi \chi^{\prime}} v & \simeq \sum_{f} \frac{2 c_{f}}{\pi} G_{F}^{2} m_{\chi}^{2} \beta^{\prime}\left[\frac{\left(g^{\prime} \cos 2 \beta / g \sin \beta\right) m_{W} m_{f} \xi_{f}}{\left[\left(m_{H_{3}^{0}}^{2}-4 m_{\chi}^{2}\right)^{2}+m_{H_{3}^{0}}^{2} \Gamma_{H_{3}^{0}}^{2}\right]^{1 / 2}}-\frac{\left(T_{3 L} \tan \theta_{W}\right) m_{W} m_{f} \zeta_{f}}{m_{0}^{2}+\beta^{\prime 2} m_{\chi}^{2}}\right]^{2}, \\
\sigma_{\chi^{\prime} \chi^{\prime}} v & \simeq \sum_{f} \frac{4 c_{f}}{\pi} G_{F}^{2} m_{\chi}^{2} \beta^{\prime}\left[\frac{\left(\xi_{f} \cot \beta / 2\right)\left(m_{\chi^{\prime}}+\mu_{H}\right) m_{f}}{\left[\left(m_{H_{3}^{0}}^{2}-4 m_{\chi}^{2}\right)^{2}+m_{H_{3}^{0}}^{2} \Gamma_{H_{3}^{0}}^{2}\right]^{1 / 2}}-\frac{m_{f}^{2} \zeta_{f}^{2} z}{4\left(m_{0}^{2}+\beta^{\prime 2} m_{\chi}^{2}\right)}\right]^{2},
\end{aligned}
$$

where $\xi_{f}=\cot \beta$ and $\zeta_{f}=\frac{1}{\sin \beta}$ for $f$ in the up sector and $\xi_{f}=\tan \beta$ for and also $\zeta_{f}=\frac{1}{\cos \beta}$ for $f$ in the down sector. The effective cross section including the coannihilation can be determined by the similar formulas to eq. (30). The dominant contributions for it are expected to come from a channel with the $t \bar{t}$ final state if $m_{\chi}>m_{t}$ is satisfied. If we focus our numerical study to the case with $m_{\chi} \simeq m_{\chi}^{\prime}$ and fix both $\tan \beta$ and $m_{H_{3}^{0}}^{2}$ to suitable values 15 , these cross sections are determined by two free parameters $m_{0}$ and $m_{\chi}$. They should satisfy some required conditions. Since $\chi$ should be lighter than the left-handed

\footnotetext{
${ }^{15}$ In this study we assume a sufficiently large value for $m_{H_{3}^{0}}^{2}$ such as $m_{H_{3}^{0}}^{2} \gg 4 m_{\chi}^{2}$, for simplicity.
} 
sneutrinos, $m_{\chi}<m_{0}$ has to be satisfied. The new contribution to the $\mu \rightarrow e \gamma$ imposes the lower bound on $m_{0}$ as found from Fig. 3. In relation to this constraint it is useful to remind that a larger $M_{3}$ allows smaller values for $m_{0}$.

Now we examine the possibility to realize the required relic abundance by these two DM. If we follow the ordinary method given in [37, 38], we can estimate the relic abundance by using the results for the effective annihilation cross section given above. Each relic abundance $\Omega_{\psi_{N_{1}}}$ and $\Omega_{\chi}$ is given by the formulas

$$
\Omega h^{2}=\frac{1.07 \times 10^{9} x_{f}}{g_{*}^{1 / 2} m_{\mathrm{pl}}(\mathrm{GeV})\left(a_{\mathrm{eff}}+3 b_{\mathrm{eff}} / x_{f}\right)}, \quad x_{f}=\ln \frac{0.038 g_{\mathrm{eff}} m_{\mathrm{pl}} m_{D M}\left(a_{\mathrm{eff}}+6 b_{\mathrm{eff}} / x_{f}\right)}{g_{*}^{1 / 2} x_{f}^{1 / 2}}
$$

where $m_{\mathrm{pl}}=1.22 \times 10^{19} \mathrm{GeV}$ and $m_{D M}$ is the mass of DM. $g_{\text {eff }}$ stands for the internal degrees of freedom of DM or the effective degrees of freedom in the coannihilation case. We can use $g_{*} \simeq 100$ as the relativistic degrees of freedom at the DM freeze-out temperature $T_{f}\left(\equiv m_{\mathrm{DM}} / x_{f}\right)$ for both $\psi_{N_{1}}$ and $\chi$.

The results are shown in Fig. 5 for the parameter set used in the previous section. In the left frame, we plot the contours for the relic abundance (29) in the $\left(M_{1}, m_{\chi}\right)$ plane for $m_{0}=0.46 \mathrm{TeV}$ (a green dotted line), $0.48 \mathrm{TeV}$ (a blue dotted line), and 0.50 TeV (a red solid line). Since we are considering that $\chi$ is the DM lighter than $\psi_{N_{1}}, M_{1}, m_{0}>m_{\chi}$ should be satisfied. The allowed regions in the $\left(M_{1}, m_{\chi}\right)$ plane are represented by the points on each contour which satisfy this condition. They are found to have the almost fixed values of $m_{\chi}$ for the larger values of $M_{1}$. The reason can be found in the right frame of Fig. 5, where the relic abundance of each DM component is plotted for the same parameters as the left frame. The same lines are used as the ones for the corresponding contours in the left frame. Since $\Omega_{\psi_{N_{1}}}$ is almost constant at large $M_{1}$ regions, the condition (29) can be satisfied only for restricted values of $\Omega_{\chi}$. Since both DM components have the same order abundance, we can expect rather different DM phenomenology from the one component DM models.

We need to note that the above solutions are obtained for rather large neutrino Yukawa couplings such as $\sqrt{\left|h_{1}\right|^{2}+\left|h_{2}\right|^{2}} \simeq 3.56$ in case of $m_{0}=0.48 \mathrm{TeV}$, for example. Although these results on the neutrino masses and the DM are interesting enough for the model, such large Yukawa couplings are dangerous for the perturbatibity and the stability of the model [17]. However, this point can be improved by considering the case $\varphi_{1}-\varphi_{2} \neq n \pi$, which makes the $s$-wave contributions to the $\psi_{N_{1}}$ annihilation cross section possible. Since 

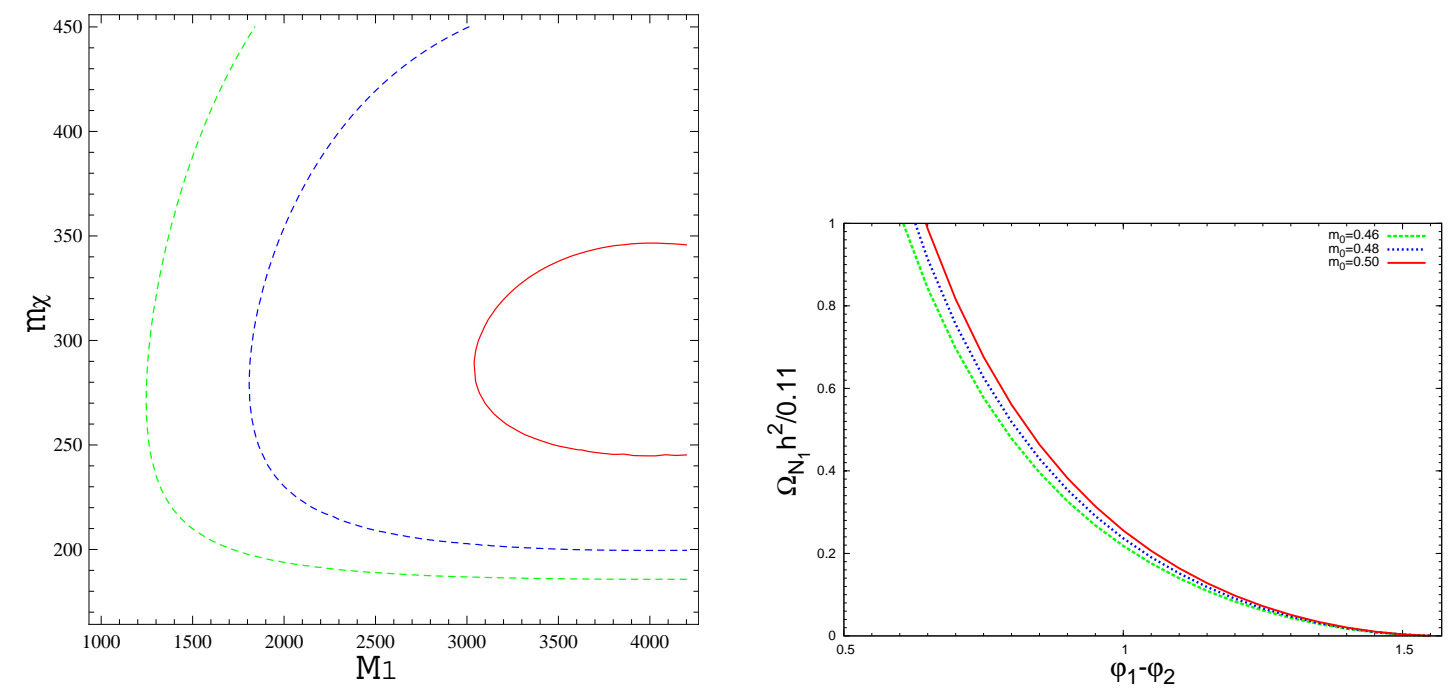

Fig. 6 The left frame is the same one as the left frame in Fig. 5. However, $\varphi_{1}-\varphi_{2}=\pi / 4$ is assumed here. The right frame shows the phase dependence of $\Omega_{N_{1}} h^{2}$ for each value of $m_{0}$ in the left frame.

the $s$-wave contributions give much larger effects than the $p$-wave ones for the thermally averaged annihilation cross section $\langle\sigma v\rangle$, the neutrino Yukawa couplings required to reduce the relic abundance of $\psi_{N_{1}}$ can be smaller. In the left frame of Fig. 6, the contours corresponding to the ones in the left frame in Fig. 5 are plotted by assuming $\left|h_{1}\right|=\left|h_{2}\right|$ and $\varphi_{1}-\varphi_{2}=\pi / 4$. The parameters are fixed to the same values as in Fig. 5 except for $\bar{\lambda}$, which is taken here as $\bar{\lambda}=8.4 \times 10^{-9}$. Since the neutrino Yukawa couplings have much smaller values like $\sqrt{\left|h_{1}\right|^{2}+\left|h_{2}\right|^{2}} \simeq 1.45$ in case of $m_{0}=0.48 \mathrm{TeV}$, the above mentioned tension is relaxed. In the right frame of Fig. 6 , the phase dependence of $\Omega_{N_{1}} h^{2}$ is shown for the same parameter setting.

\section{Probing two dark matter}

The present model has two DM components, that is, the meta stable lightest singlet fermion $\psi_{N_{1}}$ and the lightest neutralino $\chi$. They are expected to be observed by several kinds of experiments. The former one may be studied indirectly through the decay products such as charged particles and gamma rays. The anomaly reported in the cosmic rays by PAMELA and Fermi-LAT may be relevant to this decay. The latter one may be observed directly through the elastic scattering with nuclei in the same way as the ordinary lightest superparticle. However, the situation can be rather different from the 

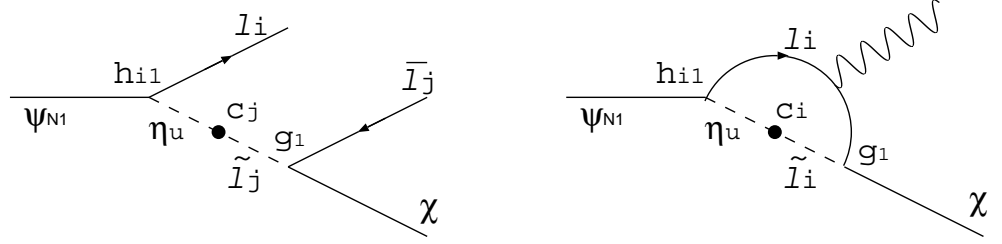

Fig. 7 Decay processes of $\psi_{N_{1}}$ to $\chi$. A bulb represents the anomaly induced interaction $c_{j} B M_{\mathrm{pl}} e^{-b_{j}} \tilde{L}_{j} \tilde{\eta}_{u}$.

MSSM since the DM relic abundance is composed of two components. In this section these subjects are briefly discussed and the detailed study will be presented elsewhere.

\subsection{Decay of the right-handed neutrino dark matter}

The relic DM in this model can be composed of the two components $\psi_{N_{1}}$ and $\chi$ as shown in the previous part. However, one of the DM candidates $\psi_{N_{1}}$ is not stable since the $Z_{2}$ symmetry which guarantees its stability is not exact. It should be reminded that this symmetry is the remnant symmetry left after the spontaneous breaking of the anomalous $\mathrm{U}(1)_{X}$. Green-Schwarz anomaly cancellation mechanism induces the $Z_{2}$ violating interaction and also the corresponding soft supersymmetry breaking term, which are shown in eqs. (9) and (10). If $\psi_{N_{1}}$ is heavier than $\chi$, this interaction brings the decay of $\psi_{N_{1}}$ to $\chi$ through the diagrams shown in Fig. 7.

We can estimate the lifetime of $\psi_{N_{1}}$ due to the decay derived by this interaction. It can be expressed as

$$
\tau_{\psi_{N_{1}}} \sim\left(\frac{1 \mathrm{TeV}}{M_{1}}\right)^{5}\left(\frac{\mu_{\eta}}{1.3 \mathrm{TeV}}\right)^{4}\left(\frac{m_{0}}{1 \mathrm{TeV}}\right)^{4}\left(\frac{1 \mathrm{TeV}}{B}\right)^{2}\left(\frac{e^{2 b_{j}}}{10^{80}}\right) \times 10^{26} \mathrm{sec}
$$

where we use $h_{i 1}^{N}=O(1)$ and $c_{j}=O(1)$. If $M_{1} \gg m_{0}$ is satisfied, $m_{0}$ should be replaced by $M_{1}$ in eq. (36). From this formula, we find that $\psi_{N_{1}}$ can have a sufficiently long lifetime compared with the age of universe as long as $b_{j}>82$ is satisfied. Thus, although the true stable DM is the lightest neutralino $\chi$, we need to take account of the contribution of $\psi_{N_{1}}$ to the relic DM abundance in the universe as discussed in the previous part.

On the other hand, depending on the scale of the $Z_{2}$ breaking $c_{j} M_{\mathrm{pl}} B e^{-b_{j}}$ in eq. (10), particles yielded in the decay of $\psi_{N_{1}}$ could bring additional contributions to the cosmic rays in the present universe. They could be detected as the anomaly in the expected flux of the cosmic rays through the various observation. In fact, if $b_{j} \sim 92$ is satisfied, this anomaly induced superweak interaction causes a large enhancement factor of $O\left(10^{80}\right)$ 

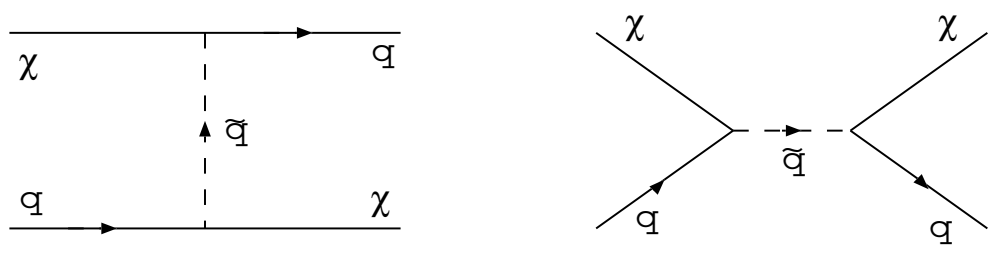

Fig. 8 Elastic scattering diagrams of the bino-like neutralino $\chi$ with the quark which are relevant to the direct search of $\chi$.

in eq. (36) to realize a long lifetime of $O\left(10^{26}\right)$ sec for $\psi_{N_{1}} \cdot 16$ This lifetime is known to be suitable to explain the charged cosmic ray anomaly reported by PAMELA [12] and Fermi-LAT [13]. Since $\psi_{N_{1}}$ couples only with leptons and sleptons because of the U(1) $X$ symmetry, this decay can yield a pair of a lepton and an antilepton, or a photon in addition to the lightest neutralino $\chi$. This feature is favored by the lepto-philic nature of the PAMELA observations. Moreover, the flavor structure of the neutrino Yukawa couplings (17) can restrict the flavor of the final charged leptons to $\mu$ and $\tau 17$ Since this makes positrons and electrons produced by their decay soft, the model becomes favorable for the explanation of a plateau at high energy regions of $e^{+}+e^{-}$spectrum found in the Fermi-LAT observations [39].

The heavier DM component $\psi_{N_{1}}$ has also a radiative decay mode to the lightest neutralino $\chi$. Its one-loop diagram is shown in Fig. 7. This decay associates a characteristic gamma which can be detected through the observation of the cosmic gamma rays. It is expected to appear as a line shape spectrum at the energy $E_{\gamma}=\left(M_{1}^{2}-m_{\chi}^{2}\right) / 2 M_{1}$, which corresponds to the endpoint of the gamma-ray spectrum caused by the bremsstrahlung and the inverse Compton scatterings associated to the charged decay products of $\psi_{N_{1}}$. This could be a clear evidence of the model.

\subsection{Direct detection of the neutralino dark matter}

Direct detection of the DM is expected to clarify the nature of DM [3]. Several experiments such as CDMSII, XENON100 and XMASS to observe its elastic scattering with nuclei are

\footnotetext{
${ }^{16} \mathrm{It}$ is interesting to note that this value of $b_{j}$ can be consistent with the value of $\operatorname{Tr} X$ for $g_{X}=O(1)$, which is required to realize the vacuum with the desired values of $\varepsilon_{ \pm}$as discussed below eq. (5) and in Appendix A.

${ }^{17}$ In that case we need to impose $c_{e}=0$ additionally in the anomaly induced interaction $c_{j} M_{\mathrm{pl}} e^{-b_{j}} L_{j} \eta_{u}$.
} 
now under going or will start in the near future. In the study of DM models, it is crucial to address the discriminative features of the model, which are expected to be shown in these experiments.

Since one DM component $\psi_{N_{1}}$ interacts with the leptons only and can not have interactions with nuclei at tree level, the scattering cross section with nuclei is heavily suppressed by the loop factor. Thus, it is difficult to detect it in these experiments. On the other hand, the neutralino DM $\chi$ can be scattered with nuclei at tree level since it has the same nature as an ordinary neutralino in the MSSM. One may consider that there is no distinction with the MSSM case. However, it should be noted that the detection rate $R$ in this model can be different from the direct detection rate $R_{\mathrm{MSSM}}$ in the MSSM even if the DM $\chi$ has the same mass and the same scattering cross section with a nucleon in two models. The detection rate is approximately defined by

$$
R \simeq \sum_{i} n_{i} \frac{\rho_{\chi}}{m_{\chi}}\left\langle\sigma_{i \chi}\right\rangle
$$

where $n_{i}$ is the number of $i$ nuclei species in the detector and $\left\langle\sigma_{i \chi}\right\rangle$ is the scattering cross section of the $\chi$ and $i$ nuclei species averaged over the relative velocity between the $\chi$ and the detector. Since the present model has two DM components, the detection rate in this model is related to the MSSM one as

$$
R=\frac{\Omega_{\chi}}{\Omega_{\psi_{N_{1}}}+\Omega_{\chi}} R_{\mathrm{MSSM}},
$$

for the fixed $m_{\chi}$ and $m_{0}$. Therefore, in the present model the parameter regions to realize the same DM detection rate can be changed from the ones in the MSSM, although the interactions of the $\chi$ with quarks are same as the MSSM neutralino. This might open a new possibility for the supersymmetry breaking parameters, which is considered not to be allowed in the MSSM case.

In order to see this aspect we consider the bino-like $\chi$, as an example. The dominant contributions to the elastic scattering of the bino-like $\chi$ with nuclei come from the squark exchange shown in Fig. 8. In the MSSM case, the allowed parameter regions relevant to the detection rate have been extensively studied. We are interested here in possible changes of the detection rate from the MSSM and also the change of the allowed region of the supersymmetry breaking parameters.

We suppose the values used in Fig. 5 for the parameters relevant to the neutrino mass generation. Eq. (38) shows that the figure of $R / R_{\mathrm{MSSM}}$ as a function of $m_{\chi}$ is the same 


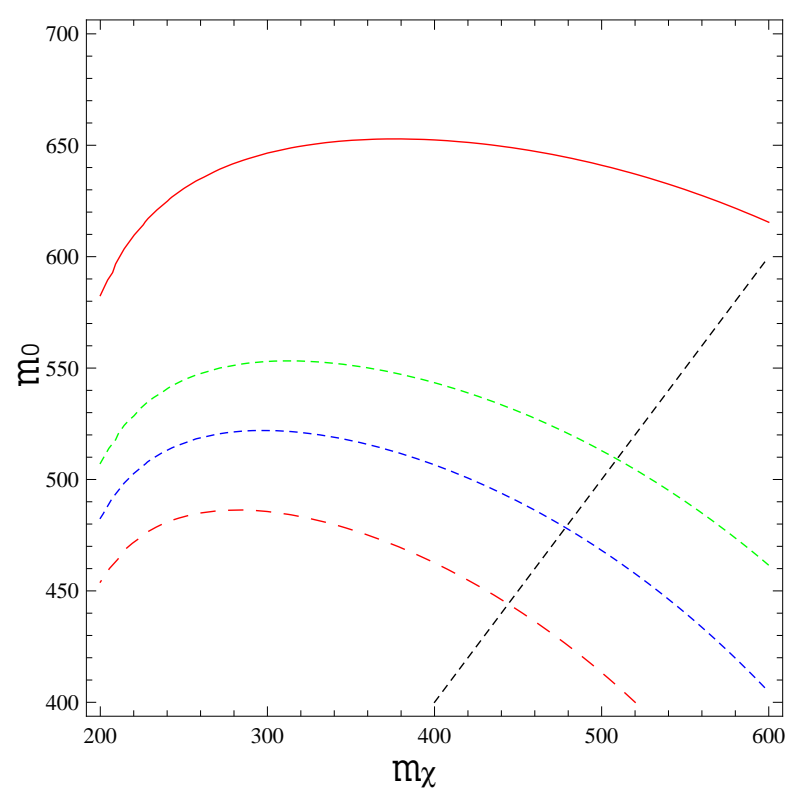

Fig. 9 The Contours of $R / R_{\mathrm{MSSM}}$ in the $\left(m_{\chi}, m_{0}\right)$ plane. Each line corresponds to $R / R_{\mathrm{MSSM}}=1$ (a red solid line), 0.6 (a green dotted line), 0.5 (a blue dotted line) and 0.4 (a red dashed line). A thin black dashed line represents a line for $m_{0}=m_{\chi}$.

as the one in the right frames of Fig. 5 for the case $\varphi_{1}-\varphi_{2}=0$. If we considered the case with $m_{0}=0.48 \mathrm{TeV}$ and $M_{1}=2.5 \mathrm{TeV}$ as an example, Fig. 5 shows that $m_{\chi}$ should be $0.2 \mathrm{TeV}$ or $0.45 \mathrm{TeV}$ to satisfy the WMAP constraint and then $R / R_{\mathrm{MSSM}} \simeq 0.5$ or 0.47 for each $m_{\chi}$ value, respectively. These suppressed detection rates compared with the MSSM are realized for much smaller $m_{0}$ values than the ones in the MSSM. The values of $\left(m_{\chi}, m_{0}\right)$ found through the direct search of the lightest neutralino are placed on the contours of $\Omega_{\chi} h^{2}$, which exist in the region where $\Omega_{\chi} h^{2}<0.11, m_{0}>m_{\chi}$ and $m_{0}>0.45 \mathrm{TeV}$. The last condition comes from the $\mu \rightarrow e \gamma$ constraint in Fig. 3. We note that the model could bring new possibilities for the detection rate of the neutralino DM and values of $\left(m_{\chi}, m_{0}\right)$ relevant to the supersymmetry breaking even if we impose the DM relic abundance constraint (see Fig. 9).

\section{Summary}

We have studied a supersymmetric model with an anomalous U(1) gauge symmetry from a view point of the neutrino masses and the DM. The model considered in this paper may be recognized as a supersymmetric extension of the radiative seesaw model for the 
neutrino masses proposed by Ma. The Froggatt-Nielsen mechanism based on the spontaneously broken anomalous U(1) symmetry generates the hierarchical structure of Yukawa couplings of the quarks and the charged leptons. Moreover, it can also explains the hierarchical couplings and mass scales required for the radiative generation of the neutrino masses. If we assume a flavor structure for the neutrino Yukawa couplings, the tri-bimaximal mixing is automatically induced.

The model has two DM components. One is stable and the other is the decaying DM. Their stability is guaranteed by two $Z_{2}$ symmetries, one of which is the ordinary $R$ parity. Since one of these discrete symmetries is assumed to be anomalous and then the $Z_{2}$ violating interaction is generated nonperturbatively, the instability of one DM component is caused. This phenomenon happens since this $Z_{2}$ symmetry is embedded into the anomalous $\mathrm{U}(1)$ gauge symmetry. Since the $Z_{2}$ violating nonpertubative interaction is extremely weak, the huge suppression factor for the decay width of this DM can be derived. As a result, its lifetime becomes longer than the age of the universe. These DM particles can be detected through both direct and indirect searches. Thus, the model may be checked through these experiments. In particular, the recent and future data coming from the cosmic ray observations might clarify the relation between this model and the physics beyond the SM.

\section{Acknowledgement}

The authors thank Mr.T. Yoshida for the collaboration at the first stage of the work. This work is partially supported by a Grant-in-Aid for Scientific Research (C) from Japan Society for Promotion of Science (No.21540262) and also a Grant-in-Aid for Scientific Research on Priority Areas from The Ministry of Education, Culture, Sports, Science and Technology (No.22011003). 


\section{Appendix A}

In this appendix we give two examples of the $\mathrm{U}(1)_{X}$ charge assignment which induce the favorable effective parameters for the generation of the mass eigenvalues and mixings of the quarks and the leptons including the neutrinos. If we define $\varepsilon_{ \pm}$as $\varepsilon_{ \pm}=\left\langle\Sigma_{ \pm}\right\rangle / M_{\mathrm{pl}}$ eq. (7) gives the expression for each parameter in the superpotential $W$ such as

$$
\begin{aligned}
& h_{i j}^{U}=y_{i j}^{U} \varepsilon_{-}^{n_{Q_{i}}+n_{U_{j}}+n_{H_{u}}}, \quad h_{i j}^{D}=y_{i j}^{D} \varepsilon_{-}^{n_{Q_{i}}+n_{D_{j}}+n_{H_{d}}}, \quad h_{i j}^{E}=y_{i j}^{E} \varepsilon_{-}^{n_{L_{i}}+n_{E_{j}}+n_{H_{d}}}, \\
& \mu_{H}=\lambda_{H} M_{\mathrm{pl}} \varepsilon_{+}^{-\frac{n_{H_{u}}+n_{H_{d}}}{n_{+}}},
\end{aligned}
$$

for the ones belonging to the MSSM. Other ones are also expressed as

$$
\begin{aligned}
& h_{i j}^{N}=y_{i j}^{N} \varepsilon_{-}^{n_{L_{i}}+n_{N_{j}}+n_{\eta_{u}}+1}, \quad \lambda_{u}=y_{\eta_{u}} \varepsilon_{+}^{-\frac{n_{\eta_{u}+n_{\phi}+n_{H_{d}}+1}}{n_{+}}}, \quad \lambda_{d}=y_{\eta_{d}} \varepsilon_{+}^{-\frac{n_{\eta_{d}}+n_{\phi}+n_{H_{u}}+1}{n_{+}}}, \\
& \mu_{\eta}=y_{\eta} M_{\mathrm{pl}} \varepsilon_{+}^{-\frac{n_{\eta_{u}+n_{\eta_{d}}+1}}{n_{+}}}, \quad M_{i j}=y_{N_{i} N_{j}} M_{\mathrm{pl}} \varepsilon_{+}^{-\frac{n_{N_{i}+n_{N_{j}}+1}}{n_{+}}}, \quad \mu_{\phi}=y_{\phi} M_{\mathrm{pl}} \varepsilon_{-}^{2 n_{\phi}+1} \text {. }
\end{aligned}
$$

In these formulas, it may be natural to suppose that the original coupling constants in the nonrenormalizable interactions, that is, $y_{i j}^{U}, \lambda_{H}$ and so on, have values of $O(1)$.

We assume that $\Sigma_{-}$and $\Sigma_{+}$obtain the VEVs defined by $\varepsilon_{-} \simeq 10^{-1}$ and $\varepsilon_{+} \simeq 10^{-4}$, respectively. The $\mathrm{U}(1)_{X}$ charge is assigned to each field as follows,

$$
\begin{array}{ll} 
& n_{Q}=(6,5,3), \quad n_{U}=(6,5,3), \quad n_{D}(4,3,3), \\
\text { example (i) } \quad & n_{L}=(9,9,9), \quad n_{E}=(1,-1,-3), \quad n_{H_{u}}=n_{H_{d}}=-6, \\
& n_{N}=(-5,-5,-5), \quad n_{\eta_{u}}=n_{\eta_{d}}=-5, \quad n_{\phi}=7, \quad n_{+}=3 .
\end{array}
$$

In this case, it is easily found that the mass matrices for the up- and down-type quarks and the charged leptons take the following form:

$$
\begin{aligned}
M_{U} & =\left(\begin{array}{ccc}
\varepsilon_{-}^{6} & \varepsilon_{-}^{5} & \varepsilon_{-}^{3} \\
\varepsilon_{-}^{5} & \varepsilon_{-}^{4} & \varepsilon_{-}^{2} \\
\varepsilon_{-}^{3} & \varepsilon_{-}^{2} & 1
\end{array}\right)\left\langle H_{u}^{0}\right\rangle, \quad M_{D}=\left(\begin{array}{ccc}
\varepsilon_{-}^{4} & \varepsilon_{-}^{3} & \varepsilon_{-} \\
\varepsilon_{-}^{3} & \varepsilon_{-}^{2} & 1 \\
\varepsilon_{-}^{3} & \varepsilon_{-}^{2} & 1
\end{array}\right)\left\langle H_{d}^{0}\right\rangle \\
M_{E} & =\left(\begin{array}{ccc}
\varepsilon_{-}^{4} & 0 & 0 \\
0 & \varepsilon_{-}^{2} & 0 \\
0 & 0 & 1
\end{array}\right)\left\langle H_{d}^{0}\right\rangle,
\end{aligned}
$$

where these $M_{f}$ are defined as $\bar{\psi}_{R} M_{f} \psi_{L}$. In the charged lepton mass matrix, the offdiagonal couplings are supposed to satisfy $y_{i j}^{E}=0($ for $i \neq j)$. From these mass matrices 
we obtain both the mass eigenvalues and the CKM matrix in the quark sector as

$$
\begin{aligned}
& m_{u}: m_{c}: m_{t}=\varepsilon_{-}^{6}: \varepsilon_{-}^{4}: 1, \quad m_{d}: m_{s}: m_{b}=\varepsilon_{-}^{4}: \varepsilon_{-}^{2}: 1, \\
& V_{u s} \sim \varepsilon_{-}, \quad V_{u b} \sim \varepsilon_{-}^{3}, \quad V_{c b} \sim \varepsilon_{-}^{2} .
\end{aligned}
$$

The charged lepton mass eigenvalues satisfy

$$
m_{e}: m_{\mu}: m_{\tau}=\varepsilon_{-}^{4}: \varepsilon_{-}^{2}: 1
$$

These can give qualitatively good results as long as $\varepsilon_{-}$takes a value of Cabibbo mixing angle 0.22 . The effective neutrino Yukawa couplings have no hierarchical structure $h_{i j}^{N}=$ $y_{i j}^{N}=O(1)$ as supposed in the text. Since the effective $\mu_{H}$ term is generated as $M_{\mathrm{pl}} \varepsilon_{+}^{4}$, it can take an appropriate value for the electroweak symmetry breaking.

Other effective parameters are estimated as

$$
\lambda_{u} \sim \lambda_{d}=O\left(\varepsilon_{+}\right), \quad M_{i}=O\left(y_{N_{i} N_{i}} M_{\mathrm{pl}} \varepsilon_{+}^{3}\right), \quad \mu_{\eta}=O\left(y_{\eta} M_{\mathrm{pl}} \varepsilon_{+}^{3}\right), \quad \mu_{\phi}=O\left(M_{\mathrm{pl}} \varepsilon_{-}^{15}\right),
$$

where we suppose that the off-diagonal couplings $y_{N_{i} N_{j}}$ are zero. These parameters are intimately related to the neutrino mass generation in the present model. The values of these parameters used in the text can be realized if $y_{N_{i} N_{i}}$ and $y_{\eta}$ have suppressed values of $O\left(10^{-2}\right)$.

In the above example, we implicitly assume that each parameter is determined by either $\varepsilon_{-}$or $\varepsilon_{+}$only but is not determined by both of them. In the next example, we consider that some of them are determined by both $\varepsilon_{-}$and $\varepsilon_{+}$. We assume that $\Sigma_{-}$and $\Sigma_{+}$obtain the VEVs defined by $\varepsilon_{-} \simeq 10^{-1}$ and $\varepsilon_{+} \simeq 4 \times 10^{-4}$ again. The $\mathrm{U}(1)_{X}$ charge is assigned to each field as follows,

$$
\begin{array}{ll} 
& n_{Q}=(6,5,3), \quad n_{U}=(3,2,0), \quad n_{D}=(5,4,4), \\
\text { example (ii) } \quad n_{L}=(7,7,7), \quad n_{E}=(4,2,0), \quad n_{H_{u}}=-3, \quad n_{H_{d}}=-7, \\
& n_{N}=(-6,-6,-6), \quad n_{\eta_{u}}=-2, \quad n_{\eta_{d}}=-9, \quad n_{\phi}=6, \quad n_{+}=3 .
\end{array}
$$

Using these charges, we can easily find what kind of factor determined by $\varepsilon_{-}$and $\varepsilon_{+}$ should appear as the lowest order one for each term in $W$. The mass matrices of quarks and charged leptons and also the neutrino Yukawa couplings $h_{i j}^{N}$ show the same features as the ones in the previous example.

The remaining effective parameters are estimated as

$$
\begin{aligned}
& \mu_{H}=O\left(M_{\mathrm{pl}} \varepsilon_{+}^{4} \varepsilon_{-}^{2}\right), \quad \lambda_{u}=O\left(\varepsilon_{+} \varepsilon_{-}\right), \quad \lambda_{d}=O\left(\varepsilon_{+}^{2} \varepsilon_{-}\right), \\
& M_{i}=O\left(M_{\mathrm{pl}} \varepsilon_{+}^{3} \varepsilon_{-}\right), \quad \mu_{\eta}=O\left(M_{\mathrm{pl}} \varepsilon_{+}^{4} \varepsilon_{-}^{2}\right), \quad \mu_{\phi}=O\left(M_{\mathrm{pl}} \varepsilon_{-}^{13}\right) .
\end{aligned}
$$


These also result in the favorable values for the parameters relevant to neutrino mass generation and also the electroweak symmetry breaking. However, it is useful to note a following point. Since $\lambda_{u} \gg \lambda_{d}$ is satisfied in this case, the formula for the dominant contribution to the neutrino masses can be changed. In fact, the term with $\lambda_{u}^{2}$ in eq. (47) could cause the similar order contribution as eq. (15) or larger contribution even if there is a suppression due to the large $\mu_{\phi}$.

\section{Appendix B}

In this Appendix we give the formulas for the contributions to the neutrino masses due to the diagrams (b) and (c) in Fig. 1. These diagrams have the scalar component and the fermion component of the chiral superfield $\phi$ as an internal line, respectively. The expressions for their contributions can be derived by taking account of eq. (14).

The neutrino mass matrix induced by the diagram (b) can be expressed as

$$
\begin{aligned}
& \left(\mathcal{M}_{\nu}\right)_{\alpha \beta}=\sum_{a= \pm} \sum_{i=1}^{3} \frac{h_{\alpha i} h_{\beta i} M_{i}\left(A+a \mu_{\phi}\right)^{2} a}{(4 \pi)^{2}} \\
& \times\left[\frac{\lambda_{d}^{2} v_{u}^{2}}{8}\left(I\left(m_{\eta+}, m_{\eta+}, M_{i}, m_{\phi a}\right)+I\left(m_{\eta-}, m_{\eta-}, M_{i}, m_{\phi a}\right)-2 I\left(m_{\eta+}, m_{\eta-}, M_{i}, m_{\phi_{a}}\right)\right)\right. \\
& +\frac{\lambda_{u}^{2} v_{d}^{2}}{8}\left(I\left(m_{\eta+}, m_{\eta+}, M_{i}, m_{\phi a}\right)+I\left(m_{\eta-}, m_{\eta-}, M_{i}, m_{\phi a}\right)+2 I\left(m_{\eta+}, m_{\eta-}, M_{i}, m_{\phi a}\right)\right) \\
& \left.+\frac{\lambda_{u} \lambda_{d} v_{u} v_{d} a}{4}\left(I\left(m_{\eta+}, m_{\eta+}, M_{i}, m_{\phi a}\right)-I\left(m_{\eta-}, m_{\eta-}, M_{i}, m_{\phi a}\right)\right)\right]
\end{aligned}
$$

where the function $I$ is defined as

$$
\begin{aligned}
I\left(m_{a}, m_{b}, m_{c}, m_{d}\right) & =\frac{m_{a}^{2} \ln m_{a}^{2}}{\left(m_{b}^{2}-m_{a}^{2}\right)\left(m_{c}^{2}-m_{a}^{2}\right)\left(m_{d}^{2}-m_{a}^{2}\right)}+\frac{m_{b}^{2} \ln m_{b}^{2}}{\left(m_{c}^{2}-m_{b}^{2}\right)\left(m_{d}^{2}-m_{b}^{2}\right)\left(m_{a}^{2}-m_{b}^{2}\right)} \\
& +\frac{m_{c}^{2} \ln m_{c}^{2}}{\left(m_{d}^{2}-m_{c}^{2}\right)\left(m_{a}^{2}-m_{c}^{2}\right)\left(m_{b}^{2}-m_{c}^{2}\right)}+\frac{m_{d}^{2}}{\left(m_{a}^{2}-m_{d}^{2}\right)\left(m_{b}^{2}-m_{d}^{2}\right)\left(m_{c}^{2}-m_{d}^{2}\right)}, \\
I\left(m_{a}, m_{a}, m_{c}, m_{d}\right) & =\frac{\left(m_{a}^{4}-m_{c}^{2} m_{d}^{2}\right) \ln m_{a}^{2}}{\left(m_{c}^{2}-m_{a}^{2}\right)^{2}\left(m_{d}^{2}-m_{a}^{2}\right)^{2}}+\frac{m_{c}^{2} \ln m_{c}^{2}}{\left(m_{d}^{2}-m_{c}^{2}\right)\left(m_{a}^{2}-m_{c}^{2}\right)^{2}} \\
& +\frac{m_{d}^{2} \ln m_{d}^{2}}{\left(m_{c}^{2}-m_{d}^{2}\right)\left(m_{a}^{2}-m_{d}^{2}\right)^{2}}-\frac{1}{\left(m_{c}^{2}-m_{a}^{2}\right)\left(m_{d}^{2}-m_{a}^{2}\right)} .
\end{aligned}
$$

On the other hand, the neutrino mass matrix induced by the diagram (c) is estimated 
as

$$
\begin{aligned}
\left(\mathcal{M}_{\nu}\right)_{\alpha \beta} & =\frac{\lambda_{d}^{2} v_{u}^{2} \mu_{\eta}^{2} \mu_{\phi}}{2(4 \pi)^{2}} \sum_{a= \pm} \sum_{i=1}^{3} h_{\alpha i} h_{\beta i} I\left(\mu_{\eta}, \mu_{\eta}, M_{i a}, \mu_{\phi}\right) \\
& -\frac{\lambda_{u}^{2} v_{d}^{2} \mu_{\phi}}{(4 \pi)^{2}} \sum_{a= \pm} \sum_{i=1}^{3} h_{\alpha i} h_{\beta i} J\left(\mu_{\eta}, M_{i a}, \mu_{\phi}\right)
\end{aligned}
$$

where $J$ is defined as

$$
\begin{aligned}
J\left(m_{a}, m_{b}, m_{c}\right) & =\left(-\frac{m_{a}^{2}}{\left(m_{b}^{2}-m_{a}^{2}\right)\left(m_{c}^{2}-m_{a}^{2}\right)}+\frac{m_{a}^{4}\left(2 m_{a}^{2}-m_{b}^{2}-m_{c}^{2}\right)}{2\left(m_{b}^{2}-m_{a}^{2}\right)^{2}\left(m_{c}^{2}-m_{a}^{2}\right)^{2}}\right) \ln m_{a}^{2} \\
& +\frac{m_{b}^{4} \ln m_{b}^{2}}{2\left(m_{c}^{2}-m_{b}^{2}\right)\left(m_{a}^{2}-m_{b}^{2}\right)^{2}}+\frac{m_{c}^{4} \ln m_{c}^{2}}{2\left(m_{b}^{2}-m_{c}^{2}\right)\left(m_{a}^{2}-m_{c}^{2}\right)^{2}} \\
& -\frac{m_{a}^{2}}{2\left(m_{b}^{2}-m_{a}^{2}\right)\left(m_{c}^{2}-m_{a}^{2}\right)} .
\end{aligned}
$$

As long as $\mu_{\phi} \gg \mu_{\eta}, M_{i}, m_{0}, A$ and $\lambda_{u} \simeq \lambda_{d}$ are satisfied, these contributions are found to be subdominant in comparison with the one induced by the diagram (a) except for the ones proportional to $\mu_{\phi}^{2}$ in eq. (47). These cause non-negligible contributions to eq. (15), which are estimated as

$$
\begin{gathered}
\sum_{i=1}^{3} \frac{h_{\alpha i} h_{\beta i} M_{i}}{(4 \pi)^{2}}\left[\frac{\lambda_{d}^{2} v_{u}^{2}}{4}\left(\tilde{I}\left(m_{\eta+}, m_{\eta+}, M_{i}\right)+\tilde{I}\left(m_{\eta-}, m_{\eta-}, M_{i}\right)-2 \tilde{I}\left(m_{\eta+}, m_{\eta-}, M_{i}\right)\right)\right. \\
\left.+\frac{\lambda_{u}^{2} v_{d}^{2}}{4}\left(\tilde{I}\left(m_{\eta+}, m_{\eta+}, M_{i}\right)+\tilde{I}\left(m_{\eta-}, m_{\eta-}, M_{i}\right)+2 \tilde{I}\left(m_{\eta+}, m_{\eta-}, M_{i}\right)\right)\right],
\end{gathered}
$$

where the function $\tilde{I}$ is defined as

$$
\begin{aligned}
\tilde{I}\left(m_{a}, m_{b}, m_{c}\right) & =\frac{m_{a}^{2} \ln m_{a}^{2}}{\left(m_{b}^{2}-m_{a}^{2}\right)\left(m_{c}^{2}-m_{a}^{2}\right)}+\frac{m_{b}^{2} \ln m_{b}^{2}}{\left(m_{c}^{2}-m_{b}^{2}\right)\left(m_{a}^{2}-m_{b}^{2}\right)}+\frac{m_{c}^{2} \ln m_{c}^{2}}{\left(m_{b}^{2}-m_{c}^{2}\right)\left(m_{a}^{2}-m_{c}^{2}\right)}, \\
\tilde{I}\left(m_{a}, m_{a}, m_{c}\right) & =\frac{m_{a}^{2}-m_{c}^{2}+m_{c}^{2} \ln \left(m_{c}^{2} / m_{a}^{2}\right)}{\left(m_{a}^{2}-m_{c}^{2}\right)^{2}} .
\end{aligned}
$$

This could be the same order contribution to the neutrino mass matrix as the one given in eq.(15). However, it is useful to note that these contributions do not change the structure of the MNS matrix but they only change the values of $\Lambda_{i}$ somewhat.

\section{Appendix C}

The $p$-wave contributions in case of $\varphi_{1} \neq \varphi_{2}+n \pi$ are given by

$$
\begin{aligned}
& p_{F}=\frac{1}{3}-\frac{M_{1}^{2}\left(3 M_{1}^{2}+5 m_{\eta a}^{2}\right)}{6\left(M_{1}^{2}+m_{\eta a}^{2}\right)^{2}}-\frac{M_{1}^{2}\left(3 M_{1}^{2}+5 m_{\eta b}^{2}\right)}{6\left(M_{1}^{2}+m_{\eta b}^{2}\right)^{2}}+\frac{M_{1}^{4}}{3\left(M_{1}^{2}+m_{\eta a}^{2}\right)\left(M_{1}^{2}+m_{\eta b}^{2}\right)}, \\
& p_{S}=-\frac{1}{2}+\frac{5}{12} \beta^{2}+\frac{M_{1}^{2} \beta^{2}\left(\mu_{\eta}^{2}+4 M_{1}^{2} \beta^{2}\right)}{3\left(\mu_{\eta}^{2}+M_{1}^{2} \beta^{2}\right)^{2}} .
\end{aligned}
$$




\section{References}

[1] WMAP Collaboration, D. N. Spergel, et al., Astrophys. J. 148 (2003) 175; SDSS Collaboration, M. Tegmark, et al., Phys. Rev. D69 (2004) 103501.

[2] SNO Collaboration, Q. R .Ahmad, et al., Phys. Rev. Lett. 89 (2002) 011301; SuperKamiokande Collaboration, Y. Fukuda, et al., Phys. Rev. Lett. 81 (1998) 1562; KamLAND Collaboration, K. Eguchi, et al., Phys. Rev. Lett. 90 (2003) 021802; K2K Collaboration, M. H. Ahn, et al., Phys. Rev. Lett. 90 (2003) 041801.

[3] For a review, see for example, G. Jungman, M. Kamionkowski and K. Griest, Phys. Rept. 267 (1996) 195; G. Bertone, D. Hooper and J. Silk, Phys. Rept. 405 (2005) 279.

[4] E. Ma, Phys. Rev. D 73 (2006) 077301.

[5] J. Kubo, E. Ma and D. Suematsu, Phys. Lett. B642 (2006) 18.

[6] J. Kubo and D. Suematsu, Phys. Lett. B643 (2006) 336.

[7] D. Suematsu, T. Toma and T. Yoshida, Phys. Rev. D79 (2009) 093004.

[8] Y. Kajiyama, J. Kubo and H. Okada, Phys. Rev. D 75 (2007) 033001; K. S. Babu and E. Ma, Int. J. Mod. Phys. A23 (2008) 1813; D. Suematsu, Eur. Phys. J. C56 (2008) 379; E. Ma and D. Suematsu, Mod. Phys. Lett. A24 (2009) 583; S. Andreas, M. H. G. Tytgat and Q. Swillens, JCAP 0904 (2009) 004.

[9] R. Barbieri, L. E. Hall and V. S. Rychkov, Phys. Rev. D74 (2006) 015007; L. Lepoz Honorez, E.Nezri, J. F. Oliver and M. H. G. Tytgat, JCAP 02 (2007) 28; M. Gustafsson, E. Lundstrom, L. Bergstrom and J. Edsjo, Phys. Rev. Lett. 99 (2007) 041301.

[10] L. M. Krauss, S. Nasri and M. Trodden, Phys. Rev. D67 (2003) 085002; D. Aristizabal Sierra, J. Kubo, D. Restrepo, D. Suematsu and O. Zepata, Phys. Rev. D79 (2009) 013011; M. Aoki, S. Kanemura and O. Seto, Phys. Rev. Lett. 102 (2009) 051805; Phys. Rev. D80 (2009) 033007; Q.-H. Cao, E. Ma and G. Shaughnessy, Phys. Lett. B673 (2009) 152; X. J. Bi, P. H. Gu, T. Li and X. Zhang, JHEP 0904 (2009) 103. 
[11] M. Lattanzi and V. W. F. Valle, Phys. Rev. Lett. 99 (2007) 121301; C. Boehm, Y. Farzan, T. Hambye, S. Palomares-Ruiz and S. Pascoli, Phys. Rev. D 77 (2008) 043516; E. Ma, Phys. Lett. B662 (2008) 49.

[12] O. Adriani et al. [PAMELA Collaboration], Nature 458 (2009) 607.

[13] A. A. Abdo et al. [The Fermi LAT Collaboration], Phys. Rev. Lett. 102 (2009) 181101.

[14] M. Beltran, D. Hooper, E. W. Kolb and Z. A. C. Krusberg, Phys. Rev. D80 (2009) 043509; V. Bager, W.-Y.Keung, D. Marfatia and G. Shaughnessy, Phys. Lett. B672 (2009) 141; I. Cholis, L. Goodenough, D. Hooper, M. Simet and N. Weiner, Phys. Rev. D80 (2009) 123511;M. Cirelli, M. Kadastik, M. Raidal and A. Strumia, Nucl. Phys. B813 (2009) 1.

[15] J. Hisano, S. Matsumoto and M. M. Nojiri, Phys. Rev. Lett. 92 (2004) 031303.

[16] D. Feldman, Z. Liu and P. Nath, Phys. Rev. D79 (2009) 063509; M. Ibe, H. Murayama, T.T. Yanagida, Phys. Rev. D79 (2009) 095009.

[17] D. Suematsu, T. Toma and T. Yoshida, Phys. Rev. D82 (2010) 013012.

[18] A. Ibarra and D. Tran, JCAP 0807 (2008) 002; JCAP 0902 (2009) 021.

[19] A. Arvanitaki, S. Dimopoulos, S. Dubovsky, P. W. Graham, R. Harnik and S. Rajendran, Phys. Rev.D79 (2009) 105022; Phys. Rev.D80 (2009) 055011; E. Nardi, F. Sannino and A. Strumia, JCAP 0901 (2009) 043; C. H. Chen, C. Q. Geng and D. V. Zhuridov, Phys. Lett. B675 (2009) 77.

[20] F. Takayama and M. Yamaguchi, Phys. Lett. B 485 (2000) 388; K. Ishiwata, S. Matsumoto and T. Moroi, Phys. Rev. D 78 (2008) 063505; S. L. Chen, R. N. Mohapatra, S. Nussinov and Y. Zhang, Phys. Lett. B677 (2009) 311.

[21] K. Ishiwata, S. Matsumoto and T. Moroi, JHEP 0905 (2009) 110; P. f. Yin, Q. Yuan, J. Liu, J. Zhang, X. j. Bi and S. h. Zhu, Phys. Rev. D 79 (2009) 023512; I. Gogoladze, R. Khalid, Q. Shafi and H. Yuksel, Phys. Rev. D79 (2009) 055019; S. Shirai, F. Takahashi and T. T. Yanagida, Phys. Lett. B680 (2009) 485. 
[22] K. Hamaguchi, S. Shirai and T. T. Yanagida, Phys. Lett. B 673 (2009) 247.

[23] E. Ma, Annales Fond. Broglie 31 (2006) 285.

[24] H. Fukuoka, J. Kubo and D. Suematsu, Phys. Lett. B678 (2009) 401.

[25] D. Suematsu, T. Toma and T. Yoshida, Int. J. Mod. Phys. A25 (2010) 4033.

[26] Q. H. Cao, E. Ma, J. Wudka and C. P. Yuan, arXiv:0711.3881; M. Fairbairn andJ. Zupan, JCAP 0907 (2009) 001.

[27] C. Froggatt and H. B. Nielsen, Phys. Lett. B147 (1979) 277.

[28] L. E. Ibanez and G. G. Ross, Phys. Lett. 332 (1994) 100; V. Jain and R. Shrock, Phys. Lett. 352 (1995) 83; P. Binetruy and P. Ramond, Phys. Lett. B350 (1995) 49; E. Dudas, S. Pokorski and C. A. Savoy, Phys. Lett. B356 (1995) 45; P. Binetruy, S. Lavignac and P. Ramond, Nucl. Phys. B477 (1996) 353; E. J. Chun and A. Lukas, Phys. Lett. B387 (1996) 99; E. Dudas, C. Grojean, S. Pokorski and C. A. Savoy, Nucl. Phys. B481 (1996) 85; K. Choi, E. J. Chun and H. D. Kim, Phys. Lett. B394 (1997) 89; P. Binetruy, N. Irges, S. Lavignac and P. Ramond, Phys. Lett. B403 (1997) 38; D. Suematsu, Phys. Rev. D64 (2001) 073013; P. H. Chankowski, K. Kowalska, S. Lavignac and S. Pokorski, Phys. Rev. D71 (2005) 055004.

[29] H. K. Dreiner, H. Murayama and M. Thormeier, Nucl. Phys. B729 (2005) 278; H. K. Dreiner, C. Luhn, H. Murayama and M. Thormeier, Nucl. Phys. B774 (2007) 127; Nucl. Phys. B795 (2008) 172; C. Coriano, M.Guzzi and A.Mariano, arXiv:1010.2010.

[30] D. A. Sierra, D. Restrepo and O. Zapata, Phys. Rev. D 80 (2009) 055010.

[31] A. Adulpravitchai, M. Lindner, A. Merle, R. N. Mohapatra, Phys. Lett. B680 (2009) 476.

[32] M. Dine, N. Seiberg and E. Witten, Nucl. Phys. B289 (1987) 589; J. Atick, L. Dixon and A. Sen, Nucl. Phys. B292 (1987) 109; M. Dine I. Ichinose and N. Seiberg, Nucl. Phys. B293 (1987) 253

[33] M. B. Green and J. H. Schwarz, Phys. Lett. B149 (1984) 117-122. 
[34] T. Banks and M. Dine, Phys. Rev. D53 (1996) 5790; N. Arkani-Hamed, M. Dine and S. P. Martin, Phys. Lett. B431 (1998) 329.

[35] M. L. Brooks et al. [MEGA Collaboration], Phys. Rev. Lett. 83 (1999) 1521; B. Aubert et al. [BABAR Collaboration], Phys. Rev. Lett. 95 (2005) 041802.

[36] G. W. Bennett et al. [Muon g-2 Collaboration], Phys. Rev. D73 (2006) 072003; K. Hagiwara, A. D. Martin, D. Nomura and T. Teubner, Phys. Lett. B649 (2007) 173.

[37] K. Griest and D. Seckel, Phys. Rev. D43 (1991) 3191.

[38] K. Griest, M. Kamionkowski and M. S. Turner, Phys. Rev. D 41 (1990) 3565; M. Drees and M. M. Nojiri, Phys. Rev. D 47 (1993) 376.

[39] P. Meade, M. Papucci, A. Strumia and T. Volansky, Nucl. Phys. B831 (2010) 178. 